TRANSACTIONS OF THE

AMERICAN MATHEMATICAL SOCIETY

Volume 349, Number 7, July 1997, Pages 2663-2685

S 0002-9947(97)01957-0

\title{
VERMA TYPE MODULES OF LEVEL ZERO FOR AFFINE LIE ALGEBRAS
}

\author{
VIATCHESLAV FUTORNY
}

\begin{abstract}
We study the structure of Verma type modules of level zero induced from non-standard Borel subalgebras of an affine Kac-Moody algebra. For such modules in "general position" we describe the unique irreducible quotients, construct a BGG type resolution and prove the BGG duality in certain categories. All results are extended to generalized Verma type modules of zero level.
\end{abstract}

\section{INTRODUCTION}

One of the significant differences between finite-dimensional and affine KacMoody algebras is the existence of root system partitions which are not "equivalent" to the standard partition into positive and negative roots. All $W \times\{ \pm 1\}$ inequivalent partitions for affine root systems ( $W$ is the Weyl group) were classified in [10], [11] and in [5], [6]. There exist only a finite number of them (always more than one) and each such partition, labeled by some finite set of integers $X$, defines a non-standard Borel subalgebra $B_{X}$ of the affine Lie algebra $\mathfrak{G}$ and a Verma type module $M_{X}(\lambda)$ induced from $B_{X}$. Verma type modules were introduced in [10] and [6]. The main difference between the classical Verma modules [12] and the modules induced from the non-standard Borel subalgebras is that the latter always have both finite and infinite-dimensional weight spaces. Verma type modules of a non-zero level, i.e. when the central element acts with a non-zero charge, were extensively studied in [1] and [9]. In this case the structure of a module $M_{X}(\lambda)$ is completely determined by its subspace $M^{f}(\lambda)$ containing all finite-dimensional weight subspaces of $M_{X}(\lambda)$. The subspace $M^{f}(\lambda)$ has a module structure for a certain infinite-dimensional Lie subalgebra $\tilde{\mathfrak{G}}^{f}$ with a triangular decomposition [13], and, when the central element acts with a non-zero charge, any submodule $N \subset M_{X}(\lambda)$ can be recovered from $N^{f}=N \cap M_{X}^{f}(\lambda)$. This leads to the equivalence between a certain category $\mathfrak{O}_{\lambda}^{X}$ of $\mathfrak{G}$-modules and a certain category of $\tilde{\mathfrak{G}}^{f}$-modules, which implies the BGG duality in $\mathfrak{D}_{\lambda}^{X}$ and a BGG type resolution for $M_{X}(\lambda)[2]$. These results were extended in [3] for the generalized Verma type modules of a non-zero level induced from a non-standard parabolic subalgebra.

Received by the editors March 27, 1995.

1991 Mathematics Subject Classification. Primary 17B67.

Key words and phrases. Affine Lie algebra, Verma type module, generalized Verma type module, BGG duality.

This work was done during the author's visit at the Department of Mathematics, Queen's University, whose generous support is greatly appreciated.

(C)1997 American Mathematical Society 
In the present paper we study the Verma type modules on which the central element acts with a zero charge. When $X=\emptyset$ such modules were considered in [10], [11], [6] and [7]. The case of a zero level is apriori more difficult than that of a nonzero level since the module $M_{X}(\lambda)$ may have subquotients that are not the quotients of Verma type modules [7]. Nevertheless, a similar approach can be developed for $\lambda$ in "general position". In particular, we describe the submodules and the irreducible quotients $L_{X}(\lambda)$ of $M_{X}(\lambda)$ when $\lambda$ is in "general position". The equivalence of suitable categories for "general position" then leads to the BGG duality in a certain category $\mathfrak{O}_{X}(\lambda)$ and to the construction of a strong BGG resolution for $L_{X}\left(\lambda_{0}\right)$, $X$ connected, $\lambda_{0}$ trivial. All results are extended for generalized Verma modules of zero level induced from a non-standard parabolic subalgebra.

Now we briefly describe the structure of the paper. In section 2 we recall the construction and the basic properties of Verma type modules and generalized Verma type modules. In section 3 we establish an important technical result (Proposition 3.2) and discuss the properties of the imaginary Verma modules $M_{\emptyset}(\lambda)$.

Since the central element of $\mathfrak{G}$ acts trivially, the module $M_{X}(\lambda)$ is reducible and can be substituted by a certain quotient $\tilde{M}_{X}(\lambda)$. The central result of section 4 is Theorem 4.8, which establishes the criterion of irreducibility for modules $\tilde{M}_{X}(\lambda)$. Section 5 is devoted to the study of modules $M_{X}(\lambda)$ under the assumption that $\tilde{M}_{X}(\lambda)$ is irreducible. In section 6 we discuss the irreducible quotients of $M_{X}(\lambda)$ (Theorem 6.1) in the particular case when $\tilde{M}_{X}^{f}(\lambda)$ is an irreducible $\tilde{\mathfrak{G}}^{f}$-module. We lift that restriction in section 7 and describe the irreducible quotients of $M_{X}(\lambda)$ for $\lambda$ in "general position" (Theorem 7.7). A strong BGG resolution for modules $L_{X}\left(\lambda_{0}\right)$ with connected $X$ and trivial $\lambda_{0}$ is constructed in section 8 (Theorem 8.2), and the BGG duality in certain categories $\mathfrak{O}_{X}(\lambda)$ of $\mathfrak{G}$-modules with $\lambda$ in "general position" is established in section 9 (Theorem 9.6). The generalized Verma type modules of level zero are discussed in section 10, and suitable categories $\mathfrak{O}_{X, S}(\lambda, q)$ with the BGG duality in section 11. Some subcategories of $\mathfrak{O}_{X, S}(\lambda, q)$ with the BGG duality are considered in section 12 .

\section{Preliminaries}

Let $\mathbb{C}$ denote the complex numbers, $\mathbb{C}^{*}=\mathbb{C} \backslash\{0\}, A=\left(a_{i j}\right), 0 \leq i, j \leq n$, be a generalized Cartan matrix of affine type and $\mathfrak{G}=\mathfrak{G}(A)$ be the corresponding affine Kac-Moody algebra of rank $n+1$ with a Cartan subalgebra $\mathfrak{H}$ and a one-dimensional centre $Z=\mathbb{C} c \subset \mathfrak{H}$. Let also $\Delta=\Delta^{r e} \cup \Delta^{i m}$ be the root system of $\mathfrak{G}$, where $\Delta^{r e}$ is the set of real roots, $\Delta^{i m}=\{k \delta \mid k \in \mathbb{Z} \backslash\{0\}\}$ is the set of imaginary roots and $\delta$ is an indivisible imaginary root. We use [12] as our main reference for Kac-Moody algebras. It follows from [5] that one can choose a basis $\pi_{0}=\left\{\alpha_{0}, \alpha_{1}, \ldots, \alpha_{n}\right\}$ of $\Delta$ such that $\delta=\sum_{i=0}^{n} k_{i} \alpha_{i}$, where $k_{0}=1$ and either $-\alpha_{0}+\delta \in \Delta$ or $\frac{1}{2}\left(-\alpha_{0}+\delta\right) \in \Delta$. Each $\alpha \in \Delta$ defines a root subspace $\mathfrak{G}_{\alpha}$, and $\mathfrak{G}=\mathfrak{H} \oplus \sum_{\alpha \in \Delta} \mathfrak{G}_{\alpha}$. Fix a basis $X_{\alpha}$ in each $\mathfrak{G}_{\alpha}, \alpha \in \Delta$. Unless otherwise stated we will always refer to this fixed basis.

For $\epsilon \subset \pi_{0}$ let $Q_{\epsilon}^{ \pm}$be the semigroup in $\mathfrak{H}^{*}$ generated by $\pm \epsilon, Q_{\epsilon}$ be the free abelian group generated by $\epsilon, \Delta(\epsilon)=Q_{\epsilon} \cap \Delta, \Delta_{ \pm}(\epsilon)=Q_{\epsilon}^{ \pm} \cap \Delta$. Set $\pi=\pi_{0} \backslash\left\{\alpha_{0}\right\}, Q=Q_{\pi_{0}}$, $\dot{Q}=Q_{\pi}, \dot{\Delta}=\Delta(\pi), \dot{\Delta}_{ \pm}=\Delta_{ \pm}(\pi)$. A subset $P \subset \Delta$ is called a partition if $P$ is closed under addition (i.e. $\alpha, \beta \in P, \alpha+\beta \in \Delta$ imply $\alpha+\beta \in P$ ),$P \cap-P=\emptyset$ and $P \cup-P=\Delta$.

Let $I=\{1,2, \ldots, n\}, X \subset I, \phi_{X}=\sum_{i \in I \backslash X} \alpha_{i}^{*}-\left(\sum_{i \in I \backslash X} k_{i}\right) \alpha_{0}^{*}$ if $X \neq I$ and $\phi_{I}=\sum_{i=0}^{n} \alpha_{i}^{*}$, where $\alpha_{i}^{*}\left(\alpha_{j}\right)=\delta_{i j}, i, j=0,1, \ldots, n$. Define $P(X)=\{\alpha \in$ 
$\left.\Delta \mid \phi_{X}(\alpha)>0\right\} \cup\left\{\alpha \in \Delta \mid \phi_{X}(\alpha)=0, \phi_{I}(\alpha)>0\right\}$. It was shown in [10] and [5] that any partition $P$ is $W \times\{ \pm 1\}$-equivalent to some $P(X)$, where $W$ is the Weyl group of $\Delta$.

We will fix $X$ throughout the paper. Note that if $X=I$ then $P(X)=\Delta_{+}\left(\pi_{0}\right)$. Let $(\cdot, \cdot)$ be the standard form on $\mathfrak{H}^{*}$ such that

$$
\frac{2\left(\alpha_{i}, \alpha_{j}\right)}{\left(\alpha_{i}, \alpha_{i}\right)}=a_{i j}, i, j=0, \ldots, n
$$

$\pi^{f}=\left\{\alpha_{i}, i \in X\right\}, \pi^{\prime}=\pi \backslash \pi^{f}, \tilde{\pi}=\left\{\alpha \in \pi^{\prime} \mid(\alpha, \beta)=0\right.$ for all $\left.\beta \in \pi^{f}\right\}, Q^{f}=$ $Q_{\pi^{f}}+\mathbb{Z} \delta, \Delta^{f}=Q^{f} \cap \Delta, \dot{\Delta}_{ \pm}^{f}=\Delta^{f} \cap \dot{\Delta}_{ \pm}, \Delta_{ \pm}^{f}=\Delta^{f} \cap( \pm P(X)), \tilde{\Delta}_{ \pm}=\Delta(\tilde{\pi}) \cap \dot{\Delta}_{ \pm}$, $\Delta_{ \pm}^{\prime}=\dot{\Delta}_{ \pm} \backslash \Delta\left(\pi^{f} \cup \tilde{\pi}\right)$, and let $Q_{ \pm}^{f}$ (resp. $Q_{ \pm}$) be the monoid generated by $\Delta_{ \pm}^{f}$ (resp. $\pm P(X))$.

For a Lie subalgebra $\mathfrak{A} \subset \mathfrak{G}, U(\mathfrak{A})$ denotes the universal enveloping algebra of $\mathfrak{A}$. Clearly, $U(\mathfrak{A})$ is a $Q$-graded algebra,

$$
U(\mathfrak{A})=\bigoplus_{\eta \in Q} U(\mathfrak{A})_{\eta} .
$$

An element $u \in U(\mathfrak{A})$ is called homogeneous if $u \in U(\mathfrak{A})_{\eta}$ for some $\eta \in Q$. We will identify $\mathfrak{G}$ with its injective image in $U(\mathfrak{G})$. If $y, y_{1}, \ldots, y_{m} \in \mathfrak{G}$ we set

$$
\left[y, y_{1}, \ldots, y_{m}\right]=\sum_{i=1}^{m} y_{1} \ldots y_{i-1}\left[y, y_{i}\right] y_{i+1} \ldots y_{m}
$$

and then define $[y, u]$ for any $u \in U(\mathfrak{G})$ by linearity.

For $\epsilon \subset \pi, \phi=\dot{\phi}+n \delta \in Q, \dot{\phi} \in \dot{Q}, n \in \mathbb{Z}$ and $0 \neq u \in U(\mathfrak{G})_{\phi}$ denote by $h t_{\epsilon}(\phi)$ the number of elements of $\pm \epsilon$ in the decomposition of $\dot{\phi}$, and let $h t_{\epsilon}(u)=h t_{\epsilon}(\phi)$, $|u|=n,\|u\|=|n|$. We also set $h t^{f}(\phi)=h t^{f}(u)=h t_{\pi^{f}}(\dot{\phi})$ and $|u|_{+}=\sum_{i=1}^{m}\left|n_{i}\right|$ if $u$ is a monomial in $U(\mathfrak{G}), u=X_{\phi_{1}+n_{1} \delta} \ldots X_{\phi_{m}+n_{m} \delta}, \phi_{i} \in \dot{Q}$.

Define $\mathfrak{G}_{X}^{ \pm}=\sum \mathfrak{G}_{ \pm \beta}, \beta \in P(X)$. Then we have an $X$-analog of the Cartan decomposition $\mathfrak{G}=\mathfrak{G}_{X}^{-} \oplus \mathfrak{H} \oplus \mathfrak{G}_{X}^{+}$. A subalgebra $B_{X}=\mathfrak{H} \oplus \mathfrak{G}_{X}^{+}$is called a nonstandard Borel subalgebra.

For $\epsilon \subset \pi^{\prime}$ consider the subalgebras $\mathfrak{G}_{ \pm}(\epsilon)=\sum \mathfrak{G}_{\beta}, \beta \in\left(Q_{\epsilon}^{ \pm}+\mathbb{Z} \delta\right) \cap \Delta^{r e}$, and let $\mathfrak{G}(\epsilon)$ be a subalgebra of $\mathfrak{G}$ generated by $\mathfrak{G}_{ \pm}(\epsilon)$. In particular, set $\tilde{\mathfrak{G}}_{ \pm}=\mathfrak{G}_{ \pm}(\tilde{\pi})$, $\tilde{\mathfrak{G}}=\mathfrak{G}(\tilde{\pi})$. Also let $\mathfrak{G}_{ \pm}^{\prime}=\sum \mathfrak{G}_{ \pm \beta}, \beta \in P(X), h t^{\prime}(\beta) \neq 0, \mathfrak{G}_{ \pm}^{f}\left(\right.$ resp. $\left.\mathfrak{G}^{f}\right)$ be a subalgebra generated by $\Delta_{ \pm}^{f} \cap \Delta^{r e}\left(\right.$ resp. $\left.\Delta^{f} \cap \Delta^{r e}\right), \mathbf{m}^{ \pm}=\sum \mathfrak{G}_{\beta}, \beta \in \Delta_{ \pm}^{f}$, $\mathbf{m}=\mathbf{m}^{-} \oplus \mathfrak{H} \oplus \mathbf{m}^{+}$and $\tilde{\mathfrak{G}}^{f}=\mathfrak{G}^{f}+\mathfrak{H}$. Clearly, $\left[\tilde{\mathfrak{G}}, \mathfrak{G}^{f}\right]=0$ and $\mathfrak{G}_{X}^{ \pm}=\mathbf{m}^{ \pm} \oplus \mathfrak{G}_{ \pm}^{\prime} \oplus \tilde{\mathfrak{G}}_{ \pm}$ If the Coxeter-Dynkin subdiagram corresponding to $X$ is connected, then $\mathfrak{G}^{f}$ is the derived algebra of an affine Lie algebra of rank $|X|+1$ with a root system $\Delta^{f}$. If $X$ is not connected then $\Delta^{f}$ does not have a basis consisting of real roots. Nevertheless, $\tilde{\mathfrak{G}}^{f}$ (resp. m) is a Lie algebra with a triangular decomposition [13] with respect to $Q$ (cf. [2], Remark 1.4) and it satisfies the conditions (T1) and (T2) of [14]. Let $X=\bigcup_{i=1}^{m} X_{i}$, and let the diagrams corresponding to each $X_{i}$ be connected. Then

$$
\mathfrak{G}^{f}=\sum_{i=1}^{m} \mathfrak{G}_{i}^{f},\left[\mathfrak{G}_{i}^{f}, \mathfrak{G}_{j}^{f}\right]=0, i \neq j, \bigcap_{i=1}^{m} \mathfrak{G}_{i}^{f}=Z
$$

and $\mathfrak{G}_{i}^{f}$ is the derived algebra of an affine Lie algebra of rank $\left|X_{i}\right|+1$ for each $i$.

Let

$$
G_{ \pm}=\sum \mathfrak{G}_{ \pm k \delta}, k \in \mathbb{Z}_{+} \backslash\{0\}, G=G_{-} \oplus Z \oplus G_{+},
$$




$$
\bar{G}=\left\{g \in G_{-} \oplus G_{+} \mid\left[g, \mathfrak{G}^{f}\right]=0\right\}, \bar{G}_{ \pm}=\bar{G} \cap G_{ \pm} .
$$

Then $G=\left(G \cap \mathfrak{G}^{f}\right) \oplus \bar{G}$ (cf. [6]), $\mathbf{m}=\tilde{\mathfrak{G}}^{f} \oplus \bar{G}, \mathbf{m}^{ \pm}=\mathfrak{G}_{ \pm}^{f} \oplus \bar{G}_{ \pm}$. Also consider the subalgebras $\mathbf{u}_{X}^{ \pm}=\sum \mathfrak{G}_{ \pm \beta}, \beta \in P(X) \backslash \Delta^{f}$, and $\mathbf{p}_{X}=\mathbf{u}_{X}^{+} \oplus \mathbf{m}$.

Let $\mathfrak{A}$ be a Lie subalgebra of $\mathfrak{G}$ and $\mathfrak{H} \subset \mathfrak{A}$. An $\mathfrak{A}$-module $V$ is called weight if

$$
V=\bigoplus_{\lambda \in \mathfrak{H}^{*}} V_{\lambda}, V_{\lambda}=\{v \in V \mid h v=\lambda(h) v \text { for all } h \in \mathfrak{H}\} .
$$

Set $P(V)=\left\{\lambda \in \mathfrak{H}^{*} \mid V_{\lambda} \neq 0\right\}$. For $\lambda, \mu \in \mathfrak{H}^{*}$, we say $\mu \leq \lambda$ if $\lambda-\mu \in Q_{+}^{f}$.

Let $\Omega$ be a subcategory of weight $\mathfrak{A}$-modules with irreducible objects $\left\{L_{t}, t \in T\right\}$ indexed by a certain subset $T \subset \mathfrak{H}^{*}$. An $\mathfrak{A}$-module $V \in \Omega$ has a local composition series [4] if for any $\mu \in P(V)$ there exist a sequence $V=V_{n} \supset \ldots \supset V_{0}=0$ of modules in $\Omega$ and a subset $J \subset\{1,2, \ldots, n\}$ such that

1. If $i \in J$, then $V_{i} / V_{i-1}=L_{t_{i}}, t_{i}-\mu \in Q_{+}$.

2. If $i \notin J$, then $\left(V_{i} / V_{i-1}\right)_{\nu}=0$ for all $\nu \in \mu+Q_{+}$.

We will denote by $\left[V: L_{t}\right]$ the multiplicity of $L_{t}$ in $V$, i.e. the number of $i$ 's in $J$ such that $t=t_{i}$.

\section{Verma AND Generalized Verma type modules}

Let $\lambda \in \mathfrak{H}^{*}$, and let $\mathbb{C} v_{\lambda}$ be the 1-dimensional $B_{X}$-module, where $\mathfrak{G}_{X}^{+} v_{\lambda}=0$ and $h v_{\lambda}=\lambda(h) v_{\lambda}$ for all $h \in \mathfrak{H}$. Consider a $\mathfrak{G}$-module

$$
M_{X}(\lambda)=U(\mathfrak{G}) \otimes_{U\left(B_{X}\right)} \mathbb{C} v_{\lambda}
$$

associated with $X$ and $\lambda$. This module is called a Verma type module of level $\lambda(c)$ [8], [9]. It is a weight module, and when $X=I$ the module $M_{X}(\lambda)$ is a usual Verma module [12]. In this case all weight subspaces are finite-dimensional. If $X \subsetneq I$, then $M_{X}(\lambda)$ possesses both finite and infinite-dimensional weight spaces, $\mu \in P\left(M_{X}(\lambda)\right)$ if and only if $\lambda-\mu \in Q_{+}, 0<\operatorname{dim} M_{X}(\lambda)_{\mu}<\infty$ if and only if $\mu \leq \lambda$. It has a unique maximal submodule, and we will denote by $L_{X}(\lambda)$ the unique irreducible quotient [6]. It follows from the construction that $M_{X}(\lambda)$ is a free $U\left(\mathfrak{G}_{X}^{-}\right)$-module.

From now on we will assume that $X \neq I$. Set $M^{f}(\lambda)=\sum_{\mu \leq \lambda} M_{X}(\lambda)_{\mu}$ and $L^{f}(\lambda)=\sum_{\mu \leq \lambda} L_{X}(\lambda)_{\mu}$. Both $M^{f}(\lambda)$ and $L^{f}(\lambda)$ are m-modules, and $L^{f}(\lambda)$ is the unique irreducible quotient of $M^{f}(\lambda)$. It follows from the construction of $M_{X}(\lambda)$ that $M^{f}(\lambda)$ is the Verma $\mathbf{m}$-module with highest weight $\lambda$ with respect to the triangular decomposition $\mathbf{m}=\mathbf{m}^{-} \oplus \mathfrak{H} \oplus \mathbf{m}^{+}$[13], and in particular it is $\mathbf{m}^{-}$-free. We can also view the modules $M^{f}(\lambda)$ and $L^{f}(\lambda)$ as $\mathbf{p}_{X}$-modules with the trivial action of $\mathbf{u}_{X}^{+}$.

When $\lambda(c) \neq 0$, the structure of $M_{X}(\lambda)$ is completely determined by $M^{f}(\lambda)$, and the irreducible quotients of Verma type modules in this case were described in [1] and [9].

Theorem $2.1([1],[9])$. Let $\lambda \in \mathfrak{H}^{*}, \lambda(c) \neq 0$. Then

$$
L_{X}(\lambda) \simeq U(\mathfrak{G}) \otimes_{U\left(\mathbf{p}_{X}\right)} L^{f}(\lambda)
$$

Suppose that $X \neq \emptyset$ and let $S \subsetneq \pi^{f}, N_{S}^{ \pm}=\sum \mathfrak{G}_{ \pm \alpha}, \alpha \in Q_{S}^{+}, \mathbf{m}_{S}=N_{S}^{-} \oplus \mathfrak{H} \oplus N_{S}^{+}$, $\mathbf{u}_{X, S}^{ \pm}=\sum\left(\mathfrak{G}_{ \pm \alpha} \cap \mathfrak{G}^{f}\right), \alpha \in \Delta_{+}^{f} \backslash Q_{S}^{+}, \mathbf{p}_{X, S}=\mathbf{m}_{S} \oplus \mathbf{u}_{X, S}^{+}, \mathfrak{H}_{S}=\left[N_{S}^{-}, N_{S}^{+}\right]$, $T_{X, S}^{ \pm}=\mathbf{u}_{X, S}^{ \pm} \oplus \mathbf{u}_{X}^{ \pm} \oplus \bar{G}_{ \pm}$and $N_{X, S}=\mathbf{m}_{S} \oplus T_{X, S}^{+}=\mathbf{p}_{X, S} \oplus \mathbf{u}_{X}^{+} \oplus \bar{G}_{+}$. Thus $\mathbf{m}_{S}$ is a finite-dimensional reductive Lie algebra, $\tilde{\mathfrak{G}}^{f}=\mathbf{p}_{X, S} \oplus \mathbf{u}_{X, S}^{-}$and $\mathfrak{G}_{X}^{+}=$ 
$\mathbf{u}_{X}^{+} \oplus \mathbf{u}_{X, S}^{+} \oplus N_{S}^{+} \oplus \bar{G}_{+}$. The subalgebra $N_{X, S}$ is called a non-standard parabolic subalgebra [3].

Let $\lambda \in \mathfrak{H}^{*}$ and $\lambda_{S}$ be the restriction of $\lambda$ to $\mathfrak{H}_{S}$. Suppose that $\lambda_{S}$ is dominant integral and consider a finite-dimensional irreducible $\mathbf{m}_{S}$-module $V_{S}(\lambda)$ with highest weight $\lambda$ (i.e. $\lambda+\alpha \notin P\left(V_{S}(\mu)\right)$ for any $\alpha \in S$ ). We can view $V_{S}(\lambda)$ as $N_{X, S}$-module with a trivial action of $T_{X, S}^{+}$and define the $\mathfrak{G}$-module

$$
M_{X, S}(\lambda)=U(\mathfrak{G}) \otimes_{U\left(N_{X, S}\right)} V_{S}(\lambda)
$$

associated with $X, S$ and $\lambda$. The module $M_{X, S}(\lambda)$ is called a generalized Verma type module. If $S=\emptyset$ then $M_{X, \emptyset}(\lambda)$ is the Verma type module associated with $X$ and $\lambda$. The properties of modules $M_{X, S}(\lambda)$ were discussed in [3]. Clearly, $M_{X, S}(\lambda)$ is a weight module, $M_{X, S}(\lambda) \simeq U\left(T_{X, S}^{-}\right) \otimes_{\mathbb{C}} V_{S}(\lambda)$ and $L_{X}(\lambda)$ is the unique irreducible quotient of $M_{X, S}(\lambda)$. Also note that $0<\operatorname{dim} M_{X, S}(\lambda)_{\mu}<\infty$ if and only if $\mu \in P\left(M_{X, S}(\lambda)\right)$ and $\mu \leq \lambda$. Consider the subspace $M_{X, S}^{f}(\lambda)=\sum M_{X, S}(\lambda)_{\mu}$, $\operatorname{dim} M_{X, S}(\lambda)_{\mu}<\infty$, which is an $\mathbf{m}$-module. We can also view $M_{X, S}^{f}(\lambda)$ as a $\mathbf{p}_{X^{-}}$ module with trivial action of $\mathbf{u}_{X}^{+}$.

Since $V_{S}(\lambda)$ is an $N_{X, S}$-module, it is a $\mathbf{p}_{X, S} \oplus \bar{G}_{+}$-module where $\mathbf{u}_{X, S}^{+}$and $\bar{G}_{+}$ act trivially.

Proposition $2.2([3]) . \quad$ 1. $M_{X, S}^{f}(\lambda) \simeq U(\mathbf{m}) \otimes_{U\left(\mathbf{p}_{X, S} \oplus \bar{G}_{+}\right)} V_{S}(\lambda)$.

2. $L^{f}(\lambda)$ is the unique irreducible quotient of $M_{X, S}^{f}(\lambda)$.

3. If $\lambda(c) \neq 0, \lambda_{S}$ is dominant integral and $N \subset M_{X, S}(\lambda)$ is a submodule, then $N \simeq U(\mathfrak{G}) \otimes_{U}\left(\mathbf{p}_{X}\right) N^{f}$, where $N^{f}=N \cap M_{X, S}^{f}(\lambda)$, and in particular, $M_{X, S}(\lambda) \simeq U(\mathfrak{G}) \otimes_{U\left(\mathbf{p}_{X}\right)} M_{X, S}^{f}(\lambda)$.

\section{IMAGinary Verma MOdUles}

In this section we discuss the properties of the modules $M_{\emptyset}(\lambda)$. Such modules were studied originally in [10], [11] and [7]. Following [7] we call them imaginary Verma modules.

First we establish the following technical lemma and proposition which will be used later.

Lemma 3.1. 1. If $\psi \in \Delta_{+}^{\prime}, h t^{\prime}(\psi)=1$ and $h t^{f}(\psi) \neq 0$, then there exists $\phi \in \Delta_{+}^{\prime}$ such that $\phi-\psi \in \dot{\Delta}_{-}^{f}$.

2. If $\psi \in \Delta_{+}^{\prime}$ (resp. $\psi \in \tilde{\Delta}_{+}$) and $h t_{\pi}(\psi) \neq 1$, then there exists $\phi \in \dot{\Delta}_{+}$such that $h t^{\prime}(\phi)=1$ (resp. $\tilde{h t}(\phi)=1$ ) and $\phi-\psi \in \dot{\Delta}_{-}$.

3. Let $\phi \in \Delta_{+}^{\prime}, h t^{f}(\phi)=0, n \in \mathbb{Z}$ and $-\phi+n \delta \in \Delta$. Then there exists $m \in \mathbb{Z}$, $m+n \neq 0$, such that $\phi+m \delta \in \Delta$ and

$$
T_{m, n}=\left[\mathfrak{G}_{\phi+m \delta}, \mathfrak{G}_{-\phi+n \delta}\right] \not \subset \bar{G} .
$$

4. Let $\phi, \psi \in \dot{\Delta}_{+}, \phi \neq \psi, n \in \mathbb{Z},-\psi+n \delta \in \Delta$ and $\phi-\psi \in \dot{\Delta}_{-}$. Then there exists $m \in \mathbb{Z}_{+}$such that $\phi \pm m \delta \in \Delta$ and $\phi-\psi+(n \pm m) \delta \in \Delta$.

5. If $\epsilon \subset \pi^{\prime}, \epsilon^{\prime} \subsetneq \epsilon, \epsilon^{\prime \prime} \subset \epsilon^{\prime}, \psi \in \Delta_{-}(\epsilon) \backslash \Delta_{-}\left(\epsilon^{\prime}\right)$ and $h t_{\epsilon^{\prime \prime}}(\psi) \neq 0$, then there exists $\phi \in \dot{\Delta}_{+}$with $h t_{\epsilon^{\prime \prime}}(\phi)=1$ and $\phi+\psi \in \Delta_{-}(\epsilon) \backslash \Delta_{-}\left(\epsilon^{\prime}\right)$.

Proof. Statements (1), (2) and (5) are obvious. Let $\phi \in \Delta_{+}^{\prime}$ and $n \in \mathbb{Z}$ as in (3). Since $\phi \notin \tilde{\Delta}$ and $h t^{f}(\phi)=0$, there exists $\beta \in \pi^{f}$ such that $\beta+\phi \in \Delta^{\prime}$ and $\beta-\phi \notin \Delta^{\prime}$. Suppose first that $\mathfrak{G}$ is a non-twisted affine Lie algebra. In this case, for any $m \in \mathbb{Z}, m+n \neq 0,\left[\mathfrak{G}_{\beta}, T_{m, n}\right] \neq 0$, which implies that $T_{m, n} \not \subset \bar{G}$. Now 
let $\mathfrak{G}$ be a twisted affine Lie algebra and $\mathfrak{G} \neq D_{4}^{(3)}$. If $n$ is even then for any even $m,\left[\mathfrak{G}_{\beta}, T_{m, n}\right] \neq 0$ and $T_{m, n} \not \subset \bar{G}$. Assume that $n$ is odd. If $\beta+\delta \in \Delta$ then for any $m \in \mathbb{Z}, m+n \neq 0,\left[\mathfrak{G}_{\beta+\delta}, T_{m, n}\right] \neq 0$, implying that $\left[\mathfrak{G}_{\beta}, T_{m, n}\right] \neq 0$ and $T_{m, n} \not \subset \bar{G}$. Let $\beta+\delta \notin \Delta$. Since $\left[\mathfrak{G}_{\beta},\left[\mathfrak{G}_{\phi+m \delta}, \mathfrak{G}_{\delta}\right]\right]=\mathfrak{G}_{\beta+\phi+(m+1) \delta} \neq 0$ for any odd $m$, we conclude that $\beta+\psi+m \delta \in \Delta$ and thus for any odd $m, m+n \neq 0$, $\left[\mathfrak{G}_{\beta}, T_{m, n}\right] \neq 0$ and $T_{m, n} \not \subset \bar{G}$. Suppose now that $\mathfrak{G}=D_{4}^{(3)}$. If $n \equiv 0(\bmod 3)$ then $\left[\mathfrak{G}_{\beta}, T_{m, n}\right] \neq 0$ for any $m \equiv 0(\bmod 3), m+n \neq 0$, and hence $T_{m, n} \not \subset \bar{G}$. Let $n \equiv 1(\bmod 3)$. Then $\phi+m \delta \in \Delta$ for any $m \equiv 2(\bmod 3)$. If $\beta+\delta \in \Delta$ then $\left[\mathfrak{G}_{\beta+\delta}, T_{m, n}\right] \neq 0$ for any $m \equiv 2(\bmod 3)$. Hence $\left[\mathfrak{G}_{\beta}, T_{m, n}\right] \neq 0$ and $T_{m, n} \not \subset \bar{G}$. Let $\beta+\delta \notin \Delta$. Then $0 \neq\left[\mathfrak{G}_{\beta},\left[\mathfrak{G}_{\phi+m \delta}, \mathfrak{G}_{\delta}\right]\right]=\left[\mathfrak{G}_{\beta+\phi+m \delta}, \mathfrak{G}_{\delta}\right]$ and $\beta+\phi+m \delta \in \Delta$ for any $m \equiv 2(\bmod 3)$. Hence, $\left[\mathfrak{G}_{\beta}, T_{m, n}\right] \neq 0$ and $T_{m, n} \not \subset \bar{G}$ for any $m \equiv 2(\bmod 3)$. The case when $n \equiv 2(\bmod 3)$ can be treated similarly. This completes the proof of (3). The proof of (4) is analogous to the proof of (3).

For $\epsilon \subset \pi^{\prime}$ and $\mu \in \mathfrak{H}^{*}$ set $\epsilon(\mu)=\{\beta \in \epsilon \mid(\beta, \mu)=0\}, \mathfrak{G}_{ \pm}(\epsilon, \mu)=\sum \mathfrak{G}_{\phi}$, $\phi \in\left(Q_{\epsilon}^{ \pm}+\mathbb{Z} \delta\right) \cap \Delta^{r e}, h t_{\epsilon \backslash \epsilon(\mu)}(\phi) \neq 0$.

Proposition 3.2. Let $V$ be a weight $\mathfrak{G}$-module, $\mu \in \mathfrak{H}^{*}, \mu(c)=0,0 \neq v_{0} \in V_{\mu}$, $\mathfrak{G}_{n \delta} v_{0}=0$ for all $n>n_{0} \geq 0, \epsilon \subset \pi^{\prime}, \mathfrak{G}_{+}(\epsilon) v_{0}=0, N=U\left(\mathfrak{G}_{-}(\epsilon)\right) v_{0}$, and let $V$ be free as $\mathfrak{G}_{-}(\epsilon, \mu)$-module. Then the following statements are equivalent:

1. $v_{0} \in U(\mathfrak{G}(\epsilon)) v$ for any non-zero $v \in N$.

2. $\beta \in \epsilon(\mu)$ implies $\mathfrak{G}_{-\beta} v_{0}=0$.

Proof. Let $\tilde{N}=U(\mathfrak{G}(\epsilon)) v$. Suppose that $\beta \in \epsilon(\mu)$ but $\mathfrak{G}_{-\beta} v_{0} \neq 0$. Since $\mathfrak{G}_{+}(\epsilon) v_{0}=$ $0, \mathfrak{G}_{+}(\epsilon) \mathfrak{G}_{-n \delta} v_{0}=0$ for any $n \in \mathbb{Z} \backslash\{0\}$ and $(\mu, \beta)=0$, we immediately conclude that $U(\mathfrak{G}(\epsilon)) \mathfrak{G}_{-\beta} v_{0} \not \supset v_{0}$ and hence (1) implies (2).

Assume now that $\mathfrak{G}_{-\beta} v_{0}=0$ for all $\beta \in \epsilon(\mu)$. If $\epsilon(\mu)=\epsilon$ then $N \simeq \mathbb{C} v_{0}$ and the statement (1) is trivial. Let $\epsilon(\mu) \neq \epsilon$ and $v$ an arbitrary non-zero element of $N$. Then $v=u v_{0}, u \in U\left(\mathfrak{G}_{-}(\epsilon)\right)$, and we may assume that $u$ is homogeneous. We divide the proof of statement (1) into several steps.

Step 1. Suppose that $h t_{\epsilon(\mu)}(u)=d>0$. Using the fact that $N$ is free over $\mathfrak{G}_{-}(\epsilon, \mu)$ and the PBW Theorem, we can write $u$ as a linear combination of the monomials

$$
X_{\phi_{1 i}+n_{1 i} \delta}^{\ell_{1 i}} \cdots X_{\phi_{s(i) i}+n_{s(i) i} \delta}^{\ell_{s(i)}} u_{i}
$$

where $u_{i} \in U\left(\mathfrak{G}_{-}(\epsilon, \mu)\right), h t_{\epsilon(\mu)}\left(u_{i}\right)=0, h t_{\epsilon \backslash \epsilon(\mu)}\left(\phi_{i j}\right) \neq 0, \phi_{i j} \in \Delta_{-}(\epsilon)$ or $\frac{1}{2} \phi_{i j} \in$ $\Delta_{-}(\epsilon)$ (the latter is possible only if $\left.\mathfrak{G}=A_{2 \ell}^{(2)}\right), 0<h t_{\epsilon(\mu)}\left(\phi_{s(i) i}\right) \leq \ldots \leq h t_{\epsilon(\mu)}\left(\phi_{1 i}\right)$ for all $i$ and $\ell_{i n}, n_{i j} \in \mathbb{Z}, \ell_{i j}>0$. We can also assume that $h t_{\epsilon(\mu)}\left(\phi_{s(1) 1}\right) \leq$ $h t_{\epsilon(\mu)}\left(\phi_{s(i) i}\right)$ for all $i$, and if $\phi_{s(1) 1}+n_{s(1) 1} \delta=\phi_{i j}+n_{i j} \delta$ then $i=s(j)$. Suppose that $\phi_{s(1) 1} \in \Delta_{-}(\epsilon)$. Then applying Lemma 3.1, (5) we can find an element $\phi \in \dot{\Delta}_{+}$ for which $h t_{\epsilon(\mu)}(\phi)=1$ and $\phi+\phi_{s(1) 1} \in \Delta_{-}(\epsilon)$. Also by Lemma 3.1, (4) one can choose $n \in \mathbb{Z}_{+}$such that $n>|u|_{+}, \phi+n \delta \in \Delta$ and $\phi+\phi_{s(1) 1}+\left(n_{s(1) 1}+n\right) \delta \in \Delta$. Since $\left[y, u_{i}\right]=0$ for $y \in \mathfrak{G}_{\phi+n \delta} \backslash\{0\}$ and for all $i$, the chosen ordering and $n$ guarantee that $[y, u]$ contains a monomial

$$
X_{\phi_{11}+n_{11} \delta}^{\ell_{11}} \ldots X_{\phi_{s(1) 1}+n_{s(1) 1} \delta}^{\ell_{s(1) 1}} X_{\phi+\phi_{s(1) 1}+\left(n_{s(1) 1}+n\right) \delta} u_{1}
$$

and thus $[y, u] \neq 0$. We conclude that $y v=y u v_{0}=[y, u] v_{0} \neq 0$ and $h t_{\epsilon(\mu)}([y, u])=$ $d-1$. If $\frac{1}{2} \phi_{s(1) 1} \in \Delta_{-}(\epsilon)$ then

$$
X_{-\frac{1}{2} \phi_{s(1) 1}} v=\left[X_{-\frac{1}{2} \phi_{s(1) 1}}, u\right] v_{0} \neq 0 \text { and } h t_{\epsilon(\mu)}\left(\left[X_{-\frac{1}{2} \phi_{s(1) 1}}, u\right]\right)<d .
$$


By induction on $d$ we find an element $z \in \mathfrak{G}_{+}(\epsilon)$ such that $z v \in N$ and $h t_{\epsilon(\mu)}(z v)=$ 0 .

We will assume that

$$
u=\sum_{i} c_{i} X_{\phi_{1 i}+n_{1 i} \delta}^{\ell_{1 i}} \ldots X_{\phi_{s(i) i}+n_{s(i) i} \delta}^{\ell_{s(i) i}}
$$

where $\sum_{j=1}^{s(i)} \phi_{j i}=\eta \in Q_{\epsilon}^{-}$for all $i$ and $h t_{\epsilon(\mu)}\left(\phi_{i j}\right)=0$ for all $i, j$.

Step 2. Suppose that $u \in \sum_{i} c_{i} X_{\beta_{1}+n_{1 i} \delta} \ldots X_{\beta_{k}+n_{k i} \delta},-\beta_{i} \in \epsilon \backslash \epsilon(\mu), i=1, \ldots k$, $\beta_{1}+\ldots+\beta_{k}=\eta, \beta_{i} \neq \beta_{j}$ if $i \neq j$. If $n_{0}=0$ then $0 \neq X_{-\beta_{1}-n_{11} \delta \ldots X_{-\beta_{k}}-n_{k 1} \delta} v \in$ $\tilde{N}$ is proportional to $v_{0}$ since $\mu\left(\left[X_{-\beta_{i}-n_{i 1} \delta}, X_{\beta_{i}+n_{i 1} \delta}\right]\right) \neq 0$ for all $i=1, \ldots, k$. Now let $n_{0}>0$. We may also assume that $n_{k 1} \leq n_{k i}$ for all $i$. Denote $y_{i}=$ $X_{\beta_{1}+n_{1 i} \delta} \ldots X_{\beta_{k-1}+n_{k-1 i} \delta}$. Then $X_{-\beta_{k}-n_{k 1} \delta} v \in \tilde{N}$ is proportional to

$$
w_{1}=\sum_{i: n_{k i}=n_{k 1}} c_{i} y_{i} v_{0}+\sum_{i: n_{k i}=n_{k 1}+1} c_{i}^{\prime} y_{i} X_{\delta} v_{0}+\ldots+\sum_{i: n_{k i}=n_{k 1}+p_{1}} c_{i}^{\prime} y_{i} X_{p_{1} \delta} v_{0},
$$

where $p_{1} \leq n_{0}$. Note that

$$
-\beta_{k}+\left(p_{1}-n_{k 1}\right) \delta \in \Delta \text { and } X_{-\beta_{k}+\left(p_{1}-n_{k 1}\right) \delta} v \in \tilde{N}
$$

is proportional to

$$
\begin{aligned}
w_{2}= & \sum_{i: n_{k i}=n_{k 1}} c_{i} y_{i} X_{p_{1} \delta} v_{0} \\
& +\sum_{i: n_{k i}=n_{k 1}+1} c_{i}^{\prime \prime} y_{i} X_{\left(p_{1}+1\right) \delta} v_{0}+\ldots+\sum_{i: n_{k i}=n_{k 1}+p_{2}} c_{i}^{\prime \prime} y_{i} X_{\left(p_{1}+p_{2}\right) \delta} v_{0},
\end{aligned}
$$

where $p_{1}+p_{2} \leq n_{0}$. Continuing this process we find $s>0$ for which $0 \neq w_{s+1}=$ $\sum_{i: n_{k i}=n_{k 1}} c_{i} y_{i} X_{\left(p_{1}+\ldots p_{s}\right) \delta} v_{0} \in \tilde{N}$ and hence $X_{\left(p_{1}+\ldots+p_{s}\right) \delta} v_{0} \in \tilde{N}$ by induction on $k$, implying that $\sum_{i: n_{k i}=n_{k 1}+p_{s}} c_{i}^{\prime \prime} y_{i} X_{\left(p_{1}+\ldots+p_{s}\right) \delta} v_{0} \in \tilde{N}$. Combining the inductions on $p_{s}$ and $s$ we conclude that $\sum_{i: n_{k i}=n_{k 1}} c_{i} y_{i} v_{0} \in \tilde{N}$, and then induction on $k$ completes the proof.

Step 3. Let $\mathfrak{G} \neq A_{2 \ell}^{(2)}, u \in U\left(\mathfrak{G}_{-}(\epsilon)\right)_{2 \beta+m \delta},-\beta \in \epsilon \backslash \epsilon(\mu)$. Then $u$ can be written in the form

$$
u=\sum_{k, n} a_{k n} X_{\beta+k \delta} X_{\beta+n \delta}
$$

where $a_{k n} \in \mathbb{C}^{*}, k+n=m, k \geq n$, and the basis of $\mathfrak{G}$ is chosen in such a way that $\left[X_{\beta+p \delta}, X_{q \delta}\right]=2 X_{\beta+(p+q) \delta}, p, q \in \mathbb{Z}, q \neq 0,\left[X_{-\beta+p \delta}, X_{\beta+q \delta}\right]=X_{(p+q) \delta}, p, q \in \mathbb{Z}$, $p+q \neq 0$. We will assume that there are at least two terms in (3.2); otherwise Step 3 can be easily reduced to Step 2. If $p>n_{0}-m$ and $-\beta+p \delta \in \Delta$, then $X_{-\beta+p \delta} v=X_{-\beta+p \delta} u v_{0}=-2\left(\sum_{k, n} a_{k n}\right) X_{\beta+(p+m) \delta} v_{0}$ and thus we can apply Step 2 if $X_{-\beta+p \delta} v \neq 0$. Suppose that $X_{-\beta+p \delta} v=0$. Since $N$ is free as $\mathfrak{G}_{-}(\epsilon, \mu)$-module, we conclude that $\sum_{k, n} a_{k n}=0$. Let $\bar{n}$ be the minimal number among all $n$ 's in $a_{k n}$ and let $n_{0}=0$. Since $(\mu, \beta) \neq 0$, it implies that $X_{-\beta-\bar{n} \delta} v$ is proportional to $X_{\beta+(m-\bar{n}) \delta} v_{0}$ and hence we can apply Step 2. Suppose now that $n_{0}>0$. Since $m-\bar{n}>\bar{n}$, an element of type

$$
X_{-\beta-(m-\bar{n}) \delta} X_{-\beta-\bar{n} \delta} v
$$

is proportional to

$$
w_{1}=v_{0}+b_{11} X_{-n_{11} \delta} X_{n_{11} \delta} v_{0}+\ldots+b_{k(1) 1} X_{-n_{k(1) 1} \delta} X_{n_{k(1) 1} \delta} v_{0} \in \tilde{N}
$$


where $b_{i 1} \in \mathbb{C}^{*}, 0<n_{i 1}<n_{i+11} \leq n_{0}$ and $X_{n_{i 1} \delta} v_{0} \neq 0$ for all $i=1, \ldots, k(1)$. Since $\beta+\left(n_{k(1) 1}+\bar{n}\right) \delta \in \Delta$, it implies that

$$
-\beta+\left(n_{k(1) 1}-\bar{n}\right) \delta \in \Delta \text { and } X_{-\beta-(m-\bar{n}) \delta} X_{-\beta+\left(n_{k(1) 1}-\bar{n}\right) \delta} v
$$

is proportional to

$$
w_{2}=X_{n_{k(1) 1} \delta} v_{0}+b_{12} X_{-n_{12} \delta} X_{n_{12} \delta} v_{0}+\ldots+b_{k_{2} 2} X_{-n_{k(2) 2} \delta} X_{n_{k(2) 2} \delta} v_{0} \in \tilde{N},
$$

where $b_{i 2} \in \mathbb{C}^{*}, n_{k(1) 1}<n_{i 2}<n_{i+12} \leq n_{0}$ and $X_{n_{i 2} \delta} v_{0} \neq 0$ for all $i=1, \ldots, k_{2}$. Continuing this procedure we find $s>0$ for which

$$
0 \neq w_{s+1}=X_{n_{k(s) s} \delta} v_{0} \in \tilde{N}, w_{s}-b_{k(s) s} X_{-n_{k(s) s} \delta} w_{s+1} \in \tilde{N}
$$

and we conclude by induction that

$$
X_{n_{k(s-1) s-1} \delta} v_{0} \in \tilde{N}, \ldots, X_{n_{k(1) 1} \delta} v_{0} \in \tilde{N},
$$

and $v_{0} \in \tilde{N}$, which completes the proof.

Step 4. Let $\mathfrak{G}=A_{2 \ell}^{(2)}, u \in U\left(\mathfrak{G}_{-}(\epsilon)\right)_{2 \beta+m \delta},-\beta \in \epsilon \backslash \epsilon(\mu)$. Then

$$
u=a_{m} X_{2 \beta+m \delta}+\sum_{k, n} a_{k n} X_{\beta+k \delta} X_{\beta+n \delta}
$$

where there are at least two terms in (3.3)

$$
\begin{gathered}
{\left[X_{\beta+m \delta}, X_{2 k \delta}\right]=2 X_{\beta+(m+2 k) \delta},} \\
{\left[X_{\beta+m \delta}, X_{(2 k+1) \delta}\right]=6 X_{\beta+(m+2 k+1) \delta},} \\
{\left[X_{-\beta+m \delta}, X_{\beta+p \delta}\right]=X_{(m+p) \delta},} \\
{\left[X_{-\beta+2 m \delta}, X_{2 \beta+(2 \ell+1) \delta}\right]=X_{\beta+(2 m+2 \ell+1) \delta}}
\end{gathered}
$$

$\left[X_{-\beta+(2 m+1) \delta}, X_{2 \beta+(2 \ell+1) \delta}\right]=-X_{\beta+2(\ell+m+1) \delta}, p, k, m, \ell \in \mathbb{Z}, k \neq 0, m+p \neq 0$

$a_{k n} \in \mathbb{C}^{*}, a_{m} \in \mathbb{C}$ and $a_{m}=0$ if $m$ is even. Let $p \in \mathbb{Z}$ and $2 p>n_{0}-m$. Then $X_{-\beta+2 p \delta} v=\left(a_{m}-2 \sum_{k-\text { even }} a_{k n}-6 \sum_{k-\text { odd }} a_{k n}\right) X_{\beta+(m+2 p) \delta} v_{0}$ and

$$
X_{-\beta+(2 p+1) \delta} v=\left(-a_{m}-6 \sum_{k-\text { even }} a_{k n}-2 \sum_{k-\text { odd }} a_{k n}\right) X_{\beta+(m+2 p+1) \delta} v_{0} .
$$

If $X_{-\beta+2 p \delta} v \neq 0$ or $X_{-\beta+(2 p+1) \delta} v \neq 0$ we can apply Step 2; otherwise

$$
a_{m}-2 \sum_{k-\text { even }} a_{k n}-6 \sum_{k-\text { odd }} a_{k n}=a_{m}+6 \sum_{k-\text { even }} a_{k n}+2 \sum_{k-\text { odd }} a_{k n}=0
$$

and one can show following the procedure in Step 3 that $v_{0} \in \tilde{N}$.

Step 5. Suppose that $\eta=2 \beta_{1}+\ldots+2 \beta_{n},-\beta_{i} \in \epsilon \backslash \epsilon(\mu), i=1, \ldots, n$, and for each $\phi_{i j}$ in (1) either $h t_{\epsilon}\left(\phi_{i j}\right)=1$ or $h t_{\epsilon}\left(\phi_{i j}\right)=2$ and $\frac{1}{2} \phi_{i j} \in \epsilon$. The proof of statement (1) in this case follows from Steps 3 and 4 using induction on $n$.

Step 6. Suppose that in (3.1) there exist $i$ and $j$ for which $h t_{\epsilon}\left(\phi_{j i}\right) \geq 2$ and $\frac{1}{2} \phi_{j i} \notin \epsilon$ or there exists $\beta \in-(\epsilon \backslash \epsilon(\mu))$ such that $\eta-3 \beta \in Q_{\epsilon}^{-}$. Using Lemma 3.1, $(2)$ and (4) one can find an element $y \in U\left(\mathfrak{G}_{+}(\epsilon)\right)$ such that $y v=y u v_{0}=u^{\prime} v_{0}$ where $u^{\prime} \in U\left(\mathfrak{G}_{-}(\epsilon)\right)$ and it has the same form as in Step 5. We leave the details to the reader. This completes the proof of the proposition. 
Now assume that $X=\emptyset$ and consider the properties of the modules $M_{\emptyset}(\lambda)$. One can easily see that $0<\operatorname{dim} M_{\emptyset}(\lambda)_{\mu}<\infty$ if and only if $\mu=\lambda-k \delta, k \in \mathbb{Z}_{+}$, which together with Theorem 2.1 implies that $M_{\emptyset}(\lambda)$ is irreducible if and only if $\lambda(c) \neq 0$ [10], [7].

Suppose that $\lambda(c)=0$ and denote $\pi(\lambda)=\{\beta \in \pi \mid(\lambda, \beta)=0\}$. Let $0 \neq v \in$ $M_{\emptyset}(\lambda)$. Then $N=U(\mathfrak{G})\left(\mathfrak{G}_{-}(\pi(\lambda)) \oplus G_{-}\right) v$ is a proper submodule of $M_{\emptyset}(\lambda)$, and we consider the $\mathfrak{G}$-module $M^{\prime}(\lambda)=M_{\emptyset}(\lambda) / N$. Clearly, $L_{\emptyset}(\lambda)$ is the unique irreducible quotient of $M^{\prime}(\lambda)$. Moreover, we have the following result.

Corollary 3.3 (cf. [10], Proposition 6.2 and [7], Theorem 1, (ii)). The module $M^{\prime}(\lambda)$ is irreducible.

Proof. Follows immediately from Proposition 3.2 with $n_{0}=0, \epsilon=\pi$ and $\mu=\lambda$.

Assume that $\pi(\lambda)=\emptyset$. Then the maximal submodule of $M_{\emptyset}(\lambda)$ is generated by $M_{1}=\sum_{n=1}^{\infty} M_{\emptyset}(\lambda)_{\lambda-n \delta}$ by Corollary 3.3. Moreover, any submodule $M \subset M_{\emptyset}(\lambda)$ is generated by $M \cap M_{1}$. A more general statement (Lemma 5.1) will be proved in section 5. Clearly, $M_{\emptyset}(\lambda)$ has a local composition series with all irreducible subquotients isomorphic to $M^{\prime}(\lambda-n \delta), n \in \mathbb{Z}_{+}$, and $\left[M_{\emptyset}(\lambda): M^{\prime}(\lambda-n \delta)\right]$ does not depend on the choice of a local composition series for any $n \in \mathbb{Z}_{+}$.

Proposition 3.4 ([7], Theorem 2). Let $\lambda \in \mathfrak{H}^{*}, \lambda(c)=0$ and $\pi(\lambda)=\emptyset$. Then $\operatorname{Hom}_{\mathfrak{G}}\left(M_{\emptyset}(\mu), M_{\emptyset}(\lambda)\right) \neq 0$ if and only if $\mu=\lambda-n \delta$ for some $n \in \mathbb{Z}_{+}$, and moreover $\operatorname{dim} \operatorname{Hom}_{\mathfrak{G}}\left(M_{\emptyset}(\lambda-n \delta), M_{\emptyset}(\lambda)\right)=\left[M_{\emptyset}(\lambda): M^{\prime}(\lambda-n \delta)\right]=\operatorname{dim} M_{\emptyset}(\lambda)_{\lambda-n \delta}$.

Remark 3.5. If $\lambda \in \mathfrak{H}^{*}, \lambda(c)=0$ and $\pi(\lambda)=\pi$, then $L_{\emptyset}(\lambda)$ is the trivial onedimensional module and the maximal submodule of $M_{\emptyset}(\lambda)$ has irreducible subquotients which are not of type $L_{\emptyset}(\mu)[7]$.

\section{Verma type modules of Zero LEVEL}

From now on we assume that $X \neq I \cup \emptyset$. In this section we begin a study of Verma type modules $M_{X}(\lambda)$ with $\lambda(c)=0$. Consider a subspace $\bar{M}=U\left(\bar{G}_{-}\right) \bar{G}_{-} v_{\lambda}$ of $M_{X}(\lambda)$.

Lemma 4.1. $U(\mathfrak{G}) \bar{M}$ is a proper submodule of $M(\lambda)$.

Proof. If $U(\mathfrak{G}) \bar{M}=M_{X}(\lambda)$ then, by the PBW Theorem, $v_{\lambda}=\sum_{i} u_{i}^{-} u_{i}^{+} X_{i} v_{\lambda}$, where $u_{i}^{-} \in U\left(\mathfrak{G}_{X}^{-}\right), u_{i}^{+} \in U\left(\mathfrak{G}_{X}^{+}\right) \mathfrak{G}_{X}^{+}, X_{i} \in U\left(\overline{\mathfrak{G}}_{-}\right) \bar{G}_{-}$. But $\left[\mathfrak{G}_{X}^{+}, \bar{G}\right] \subset \mathfrak{G}_{X}^{+} \oplus Z$. Thus $v_{\lambda}=\sum_{i} u_{i}^{-} X_{i} u_{i}^{+} v_{\lambda}+\sum_{i} u_{i}^{-}\left[u_{i}^{+}, X_{i}\right] v_{\lambda}=0$, and this contradiction completes the proof.

Let $\nu: U(\mathfrak{G}) \rightarrow U(\mathfrak{G}) / U(\mathfrak{G}) \mathfrak{G}_{X}^{+}$be the natural map. We will identify the elements of $U\left(\mathfrak{G}_{X}^{-}\right)$with their images under $\nu$. Also consider the natural map $\tilde{\nu}: U(\mathfrak{G}) \rightarrow U(\mathfrak{G}) / U(\mathfrak{G})\left(\mathfrak{G}_{X}^{+} \oplus \bar{G}_{-}\right)$and the following decomposition of $U\left(\mathfrak{G}_{X}^{-}\right)$: $U\left(\mathfrak{G}_{X}^{-}\right) \simeq U\left(\mathfrak{G}_{-}^{\prime}\right) \otimes_{\mathbb{C}} U\left(\mathbf{m}^{-}\right) \otimes_{\mathbb{C}} U\left(\tilde{\mathfrak{G}}_{-}\right)$. Then $\tilde{\nu}\left(U\left(\mathfrak{G}_{X}^{-}\right)\right)=U\left(\mathfrak{G}_{X}^{-}\right) / U\left(\mathfrak{G}_{X}^{-}\right) \bar{G}_{-} \simeq$ $U\left(\mathfrak{G}_{-}^{\prime}\right) \otimes_{\mathbb{C}} U\left(\mathfrak{G}_{-}^{f}\right) \otimes_{\mathbb{C}} U\left(\tilde{\mathfrak{G}}_{-}\right)$.

Denote $\tilde{M}(\lambda)=M_{X}(\lambda) / U(\mathfrak{G}) \bar{M}$. Then $\tilde{M}(\lambda) \simeq U\left(\mathfrak{G}_{-}^{\prime}\right) \otimes_{\mathbb{C}} U\left(\mathfrak{G}_{-}^{f}\right) \otimes_{\mathbb{C}} U\left(\tilde{\mathfrak{G}}_{-}\right)$ as a vector space. Let $\tilde{M}^{f}(\lambda)=\sum \tilde{M}(\lambda)_{\mu}, \operatorname{dim} \tilde{M}(\lambda)_{\mu}<\infty$. Clearly, $\tilde{M}(\lambda) \simeq$ $M^{f}(\lambda) / U\left(\mathfrak{G}^{f}\right) \bar{M}$, and hence it is a Verma $\tilde{\mathfrak{G}}^{f}$-module with highest weight $\lambda$ with respect to the triangular decomposition $\tilde{\mathfrak{G}}^{f}=\mathfrak{G}_{-}^{f} \oplus \mathfrak{H} \oplus \mathfrak{G}_{+}^{f}$ [13]. In particular, $\tilde{M}^{f}(\lambda) \simeq U\left(\mathfrak{G}_{-}^{f}\right)$ as a vector space. Consider the natural map $\tau: M_{X}(\lambda) \rightarrow \tilde{M}(\lambda)$ and let $\tilde{v}_{\lambda}=\tau\left(v_{\lambda}\right)$. 
Lemma 4.2. Let $0 \neq v_{0} \in \tilde{M}^{f}(\lambda)_{\mu}, \mu \in H^{*}$. A $\tilde{\mathfrak{G}}$-module $V=U(\tilde{\mathfrak{G}}) v_{0}$ is irreducible if and only if $(\mu, \beta) \neq 0$ for all $\beta \in \tilde{\pi}$.

Proof. Since $\tilde{\mathfrak{G}}_{+} v_{0}=\bar{G} v_{0}=0$, then $V$ is isomorphic to $U\left(\tilde{\mathfrak{G}}_{-}\right)$as a vector space, and the lemma follows from Proposition 3.2 if we let $\epsilon=\tilde{\pi}$ and $n_{0}=0$.

Lemma 4.3. Let $0 \neq u \in U\left(\mathfrak{G}_{X}^{-}\right)$, $u$ homogeneous, and $h t^{\prime}(u)>1$. Then there exist $\phi \in \Delta_{+}^{\prime}, n \in \mathbb{Z}_{+}$and $y \in \mathfrak{G}_{\phi-n \delta}$ such that $\tilde{\nu}(y u) \neq 0$ and $h t^{\prime}(\tilde{\nu}(y u)) \neq 0$.

Proof. Using the PBW Theorem we can write

$$
u=\sum_{k} X_{-\phi_{1 k}+n_{1 k} \delta}^{\ell_{1 k}} \cdots X_{-\phi_{s(k) k}+n_{s(k) k} \delta}^{\ell_{s(k) k}} u_{k},
$$

where $h t^{\prime}\left(\phi_{i k}\right) \neq 0$ for all $i, k,-\phi_{i k}+n_{i k} \delta \neq-\phi_{j k}+n_{j k} \delta$ if $i \neq j$ for all $k ; n_{i k}$, $\ell_{i k} \in \mathbb{Z}, \ell_{i k}>0$, and $u_{k} \in U\left(\mathfrak{G}_{-}^{f} \oplus \tilde{\mathfrak{G}}_{-}\right)$. By the assumption, $\sum_{i} h t^{\prime}\left(\phi_{i k}\right)=h t^{\prime}(u)$ for each $k$. Consider a subset $\Omega \subset\left\{\phi_{i k}\right\}$ consisting of all $\psi$ such that $h t^{\prime}(\psi)=$ $\min _{i, k} h t^{\prime}\left(\phi_{i k}\right)$. We may assume that $\phi_{s(1) 1} \in \Omega, h t^{\prime}\left(\phi_{1 k}\right) \geq \ldots \geq h t^{\prime}\left(\phi_{s(k) k}\right)$ for all $k$, and that $-\phi_{s(1) 1}+n_{s(1) 1} \delta=-\phi_{i k}+n_{i k} \delta$ implies $i=s(k)$. If $\phi_{s(1) 1} \in \Delta$ then by Lemma 3.1, (2) there exists $\phi \in \Delta_{+}^{\prime}$ such that $h t^{\prime}(\phi)=1$ and $\phi-\phi_{s(1) 1} \in \dot{\Delta}_{-}$. By Lemma 3.1, (4), we can choose sufficiently large $n \in \mathbb{Z}_{+}$for which $\phi-\phi_{s(1) 1}+$ $\left(n_{s(1) 1}-n\right) \delta \in-P(X), n>\left|n_{i k}\right|$ for all $i, k$ and $n>\left|u_{k}\right|_{+}$for all $k$. For $0 \neq y \in$ $\mathfrak{G}_{\phi-n \delta}$ it follows that $\tilde{\nu}\left(y X_{-\phi_{s(1) 1}+n_{s(1) 1} \delta} \neq 0, \tilde{\nu}(y u) \neq 0\right.$ and $h t^{\prime}(\tilde{\nu}(y u)) \neq 0$. If $\frac{1}{2} \phi_{s(1) 1} \in \Delta$ then $\tilde{\nu}\left(X_{\frac{1}{2} \phi_{s(1) 1}} u\right) \neq 0$, which completes the proof.

Lemma 4.4. Let $\psi \in \Delta_{+}^{\prime}, h t^{\prime}(\psi)=1, n \in \mathbb{Z}$ and $0 \neq X_{-\psi+n \delta} \in \mathfrak{G}_{-\psi+n \delta}$. Then there exists $y \in U(\mathfrak{G})$ such that $0 \neq \tilde{\nu}\left(y X_{-\psi+n \delta}\right) \in U\left(\mathfrak{G}_{-}^{f}\right)$.

Proof. If $h t^{f}(\psi)=0$ then the statement follows from Lemma 3.1, (3). Let $h t^{f}(\psi) \neq$ 0 . By Lemma 3.1, (1) and (4) there exist $\phi \in \Delta_{+}^{\prime}$ for which $\phi-\psi \in \dot{\Delta}_{-}^{f}$, and $t \in \mathbb{Z}_{+}$ such that $\phi-\psi+(n-t) \delta \in-P(X)$ and $\phi-t \delta \in \Delta$. Then for $0 \neq y \in \mathfrak{G}_{\phi-m \delta}$ we have $0 \neq \tilde{\nu}\left(y X_{-\psi+n \delta}\right) \in U\left(\mathfrak{G}_{-}^{f}\right)$, and the lemma follows.

Proposition 4.5. Let $0 \neq u \in U\left(\mathfrak{G}_{X}^{-}\right)$, u homogeneous, $h t^{\prime}(u) \neq 0$ and $\tilde{\nu}(u) \neq 0$. Then there exists $y \in U(\mathfrak{G})$ such that $0 \neq \tilde{\nu}(y u) \in U\left(\mathfrak{G}_{-}^{f}\right) \otimes_{\mathbb{C}} U\left(\tilde{\mathfrak{G}}_{-}\right)$.

Proof. Let $u=\sum_{k} X_{-\phi_{1 k}+n_{1 k} \delta}^{\ell_{i k}} \ldots X_{-\phi_{s(k) k}+n_{s(k) k} \delta}^{\ell_{s(k) k}} u_{k}+u_{0}$, where $h t^{\prime}\left(\phi_{i k}\right) \neq 0$ for all $i, k, \phi_{i k}$ are ordered as in the proof of Lemma 4.3, $\ell_{i k}, n_{i k} \in \mathbb{Z}, \ell_{i k}>0$, $u_{k} \in U\left(\mathfrak{G}_{-}^{f}\right) \otimes_{\mathbb{C}} U\left(\tilde{\mathfrak{G}}_{-}\right)$and $u_{0} \in U(\mathfrak{G}) \bar{G}_{-}$. Suppose that $h t^{\prime}(u)=\sum_{i} h t^{\prime}\left(\phi_{i k}\right)=r$. Using Lemmas 4.3 and 4.4 and induction on $r$, we can choose $\psi_{1}, \ldots, \psi_{r} \in \Delta_{+}^{\prime}$ and sufficiently large $t_{1}, \ldots, t_{r}$ such that $0 \neq \tilde{\nu}\left(y_{r} \ldots y_{1} u\right) \in U\left(\mathfrak{G}_{-}^{f}\right) \otimes_{\mathbb{C}} U\left(\tilde{\mathfrak{G}}_{-}\right)$, where $0 \neq y_{i} \in \mathfrak{G}_{\psi_{i}-t_{i} \delta}, i=1, \ldots, r$.

Remark 4.6. Proposition 4.5 is also valid in the case when the element $u$ is not homogeneous, i.e., $u=\sum_{i} c_{i} u_{i}$ where $u_{i} \in U\left(\mathfrak{G}_{X}^{-}\right)_{\eta_{i}}, \eta_{i} \in Q$ and $\eta_{i} \neq \eta_{j}$ if $i \neq j$.

Proposition 4.7. Let $0 \neq v \in \tilde{M}(\lambda)_{\mu}, \mu \in \mathfrak{H}^{*}$, and $(\lambda, \alpha) \neq 0$ for all $\alpha \in \tilde{\pi}$. Then there exists $y \in U(\mathfrak{G})$ such that $0 \neq y v \in \tilde{M}^{f}(\lambda)$.

Proof. Let $v=u \tilde{v}_{\lambda}$ where $u \in U\left(\mathfrak{G}_{X}^{-}\right)_{\mu-\lambda}$. By Proposition 4.5 there exists $y^{\prime} \in$ $U(\mathfrak{G})$ such that $0 \neq \tilde{\nu}\left(y^{\prime} u\right) \in U\left(\mathfrak{G}_{-}^{f}\right) \otimes U\left(\tilde{\mathfrak{G}}_{-}\right)$and thus $y^{\prime} v=y^{\prime} u \tilde{v}_{\lambda}=\tilde{\nu}\left(y^{\prime} u\right) \tilde{v}_{\lambda} \neq 0$. 
Using the PBW Theorem we can write $\tilde{\nu}\left(y^{\prime} u\right)=\sum_{k} u_{k}^{(1)} u_{k}^{(2)}$, where $u_{k}^{(1)} \in U\left(\mathfrak{G}_{-}^{f}\right)$ and $u_{k}^{(2)}$ are the monomials in $U\left(\tilde{\mathfrak{G}}_{-}\right)$. Let

$$
u_{k}^{(2)}=X_{-\phi_{1 k}+n_{1 k} \delta}^{\ell_{1 k}} \cdots X_{-\phi_{S(k) k}+n_{s(k) k} \delta}^{\ell_{s(k) k}}
$$

where $\phi_{i k} \in Q_{\tilde{\pi}}^{+}, n_{i j}, \ell_{i j} \in \mathbb{Z}, \ell_{i j}>0$. Among all monomials $u_{k}^{(2)}$ consider those with smallest $\left|u_{k}^{(2)}\right|$ and denote them by $\tilde{u}_{k}^{(2)}$. Thus $\tilde{\nu}\left(y^{\prime} u\right)=\sum_{n} u_{n}^{(1)} \tilde{u}_{n}^{(2)}+\sum_{t} u_{t}^{(1)} u_{t}^{(2)}$. Note that $\tilde{h} t\left(\tilde{u}_{n}^{(2)}\right)=\tilde{h} t\left(u_{t}^{(2)}\right)$ for all $n, t$ and $\tilde{u}_{n}^{(2)} \in U\left(\tilde{\mathfrak{G}}_{-}\right)_{\eta}$ for a single $\eta \in Q$ for all $n$. By Lemma 4.2 there exists $z \in U(\tilde{\mathfrak{G}})_{-\eta}$ such that $z\left(\sum_{n} \tilde{u}_{n}^{(2)}\right) \tilde{v}_{\lambda} \in \mathbb{C}^{*} \tilde{v}_{\lambda}$ and hence $0 \neq z\left(\sum_{n} u_{n}^{(1)} \tilde{u}_{n}^{(2)}\right) v_{\lambda} \in \tilde{M}^{f}(\lambda)$. Since $z \tilde{v}_{\lambda}=0$ and $\nu\left(z\left(\sum_{t} u_{t}^{(1)} u_{t}^{(2)}\right)\right) \in$ $U\left(\bar{G}_{+}\right) \bar{G}_{+}$, this implies that $0 \neq z y^{\prime} v=z y^{\prime} u \tilde{v}_{\lambda}=z \tilde{\nu}\left(y^{\prime} u\right) \tilde{v}_{\lambda}=z\left(\sum_{n} u_{n}^{(1)} \tilde{u}_{n}^{(2)}\right) \tilde{v}_{\lambda} \in$ $\tilde{M}^{f}(\lambda)$. We complete the proof by setting $y=z y^{\prime}$.

Now we are in a position to prove the criterion of irreducibility for modules $\tilde{M}(\lambda)$.

Theorem 4.8. Let $\lambda(c)=0$. The module $\tilde{M}(\lambda)$ is irreducible if and only if the following two conditions hold:

1. $\tilde{M}^{f}(\lambda)$ is an irreducible $\mathfrak{G}^{f}$-module.

2. $(\lambda, \alpha) \neq 0$ for all $\alpha \in \tilde{\pi}$.

Proof. Assume $\tilde{M}(\lambda)$ is an irreducible $\mathfrak{G}$-module. If $N^{f}$ is a proper $\mathfrak{G}^{f}$-submodule of $\tilde{M}^{f}(\lambda)$ then $U(\mathfrak{G}) N^{f}$ is a proper $\mathfrak{G}$-submodule of $\tilde{M}(\lambda)$. Thus $N^{f}=0$ and $\tilde{M}^{f}(\lambda)$ is an irreducible $\mathfrak{G}^{f}$-module. Suppose that $(\lambda, \alpha)=0$ for some $\alpha \in \tilde{\pi}$. Since $\left[\mathfrak{G}_{\alpha+n \delta}, \mathfrak{G}_{-\alpha}\right] \subset \bar{G}$ for any $n \in \mathbb{Z} \backslash\{0\}$ and $\left[\mathfrak{G}_{\alpha}, \mathfrak{G}_{-\alpha}\right] \tilde{v}_{\lambda}=0$, we have that $U(\mathfrak{G}) \mathfrak{G}_{-\alpha} \tilde{v}_{\lambda}$ is a proper $\mathfrak{G}$-submodule of $\tilde{M}(\lambda)$, which again contradicts the irreducibility of $\tilde{M}(\lambda)$.

Conversely, suppose that conditions (1) and (2) of the theorem are satisfied. Let $N$ be a non-zero $\mathfrak{G}$-submodule of $\tilde{M}(\lambda)$ and $0 \neq v \in N$. Then by Proposition 4.7 there exists $y \in U(\mathfrak{G})$ such that $0 \neq y v \in \tilde{M}^{f}(\lambda)$. Since $\tilde{M}^{f}(\lambda)$ is an irreducible $\mathfrak{G}^{f}$-module, yv generates $\tilde{M}(\lambda)$. Thus, $N=\tilde{M}(\lambda)$ and $\tilde{M}(\lambda)$ is irreducible.

\section{Structure of modules $M_{X}(\lambda)$ When $\tilde{M}(\lambda)$ is irreducible}

Let $\lambda \in \mathfrak{H}^{*}, \lambda(c)=0$. In this section we will assume that $\tilde{M}(\lambda)$ is an irreducible $\mathfrak{G}$-module, i.e. it satifies the conditions (1) and (2) of Theorem 4.8. If $N$ is a submodule of $M_{X}(\lambda)$ we will denote $[N]=N \cap \bar{M}$.

Lemma 5.1. Suppose that $\tilde{M}(\lambda)$ is an irreducible $\mathfrak{G}$-module. Then for every nonzero submodule $N$ of $M_{X}(\lambda),[N] \neq 0$ and $N$ is generated by $[N]$.

Proof. Let $N$ be a non-zero proper submodule of $M_{X}(\lambda)$ and $0 \neq v \in N$. Then $v=u v_{\lambda}$ for some $u \in U\left(\mathfrak{G}_{X}^{-}\right)$. Using the PBW Theorem we can write $u$ as the following linear combination:

$$
u=\sum_{k \in K_{0}} a_{k} u_{k}^{(1)} u_{k}^{(2)} u_{k}^{(3)} u_{k}^{(4)}
$$

where $a_{k} \in \mathbb{C}^{*}$ and $u_{k}^{(i)}$ are monomials such that $u_{k}^{(1)} \in U\left(\mathfrak{G}_{-}^{\prime}\right), u_{k}^{(2)} \in U\left(\mathfrak{G}_{-}^{f}\right)$, $u_{k}^{(3)} \in U\left(\tilde{\mathfrak{G}}_{-}\right), u_{k}^{(4)} \in U\left(\bar{G}_{-}\right)$. We will also assume that $u$ is homogeneous. If 
$u_{k}^{(4)}=0$ for at least one $k \in K_{0}$, then $\tilde{\nu}(u) \neq 0$ and by Theorem $4.8 \tilde{M}(\lambda) \simeq \tau(N)$ where $\tau: M_{X}(\lambda) \rightarrow \tilde{M}(\lambda)$. Thus $v_{\lambda} \in N$ and $N=M$, which contradicts our assumption. Therefore we can assume that $u_{k}^{(4)} \neq 0$ for all $k \in K_{0}$. Denote by $\ell(u)$ the number of different $u_{k}^{(4)}, k \in K_{0}$, in (5.1). Suppose that $\ell(u)=1$. Then

$$
u=\left(\sum_{k \in K_{0}} a_{k} u_{k}^{(1)} u_{k}^{(2)} u_{k}^{(3)}\right) u^{(4)} .
$$

Consider an element $u^{\prime}=\sum_{k \in K_{0}} a_{k} u_{k}^{(1)} u_{k}^{(2)} u_{k}^{(3)} \in U\left(\mathfrak{G}_{-}^{\prime} \oplus \mathfrak{G}_{-}^{f} \oplus \tilde{\mathfrak{G}}_{-}\right)$. By Theorem 4.8 there exists $y \in U\left(\mathfrak{G}_{X}^{f}\right)$ such that $y u^{\prime} v_{\lambda}=\nu\left(y u^{\prime}\right) v_{\lambda} \in \mathbb{C}^{*} v_{\lambda}$, and hence $\nu\left(y u^{\prime}\right) \in S(\mathfrak{H})$. Then we have $y u v_{\lambda}=y u^{\prime} u^{(4)} v_{\lambda}=u^{(4)} \nu\left(y u^{\prime}\right) v_{\lambda} \in \mathbb{C}^{*} u^{(4)} v_{\lambda}$, which implies $u^{(4)} v_{\lambda} \in[N]$ and $v \in U(\mathfrak{G})[N]$. We conclude that $N=U(\mathfrak{G})[N]$.

Suppose now that $\ell(u)>1$. Applying the same procedure as in the proof of Proposition 4.5 (see also Remark 4.6) we find an element $y_{1} \in u(\mathfrak{G})$ such that $y_{1} u v_{\lambda}=\left(\sum_{m} \tilde{a}_{m} \tilde{u}_{m}^{(2)} \tilde{u}_{m}^{(3)} \tilde{u}_{m}^{(4)}\right) v_{\lambda} \neq 0$, where $\tilde{u}_{m}^{(2)} \in U\left(\mathfrak{G}_{-}^{f}\right), \tilde{u}_{m}^{(3)} \in U\left(\tilde{\mathfrak{G}}_{-}\right), \tilde{u}_{m}^{(4)} \in$ $\mathfrak{U}\left(\bar{G}_{-}\right)$and for each $m$ there exists $k \in K_{0}$ such that $\tilde{u}_{m}^{(4)}=u_{k}^{(4)}$. Note that $\left(\sum_{m} \tilde{a}_{m} \tilde{u}_{m}^{(2)} \tilde{u}_{m}^{(3)}\right) v_{\lambda} \in \tilde{M}^{f}(\lambda)$.

Let $u_{m}^{2,3}=\tilde{u}_{m}^{(2)} \tilde{u}_{m}^{(3)}$ for all $m$ and $d=\max _{m}\left\|u_{m}^{2,3}\right\|$. Consider the element

$$
w=\sum_{m} \tilde{a}_{m} \tilde{u}_{m}^{(2)} \tilde{u}_{m}^{(3)}=\sum_{m:\left\|u_{m}^{2,3}\right\|=d} \tilde{a}_{m} u_{m}^{2,3}+\sum_{m:\left\|u_{m}^{2,3}\right\| \neq d} \tilde{a}_{m} u_{m}^{2,3} .
$$

Since $M_{X}^{f}(\lambda)$ is irreducible by Theorem 4.8, there exists an element $y_{2} \in U\left(\mathfrak{G}_{+}^{f}\right)$ such that

$$
y_{2}\left(\sum_{m:\left\|u_{m}^{2,3}\right\|=d} \tilde{a}_{m} u_{m}^{2,3}\right) v_{\lambda} \in \mathbb{C}^{*} v_{\lambda} .
$$

Also, note that $y_{2}\left(\sum_{m:\left\|u_{m}^{2,3}\right\| \neq d} \tilde{a}_{m} u_{m}^{2,3}\right) v_{\lambda}=0$. Thus $y_{2} w \in \mathbb{C}^{*} v_{\lambda}$ and

$$
y_{2} y_{1} v=y_{2} y_{1} u v_{\lambda}=\left(\sum_{\ell \in \mathfrak{L}} b_{\ell} \tilde{\tilde{u}}_{\ell}\right) v_{\lambda} \neq 0,
$$

where for each $\ell \in \mathfrak{L}$ there exists $k \in K_{0}$ such that $\tilde{\tilde{u}}_{\ell}=u_{k}^{(4)}$ and $\tilde{\tilde{u}}_{m} \neq \tilde{\tilde{u}}_{n}$ if $m \neq n$. We conclude that $0 \neq y_{2} y_{1} v \in N \cap \bar{M}$, and hence $[N] \neq 0$.

Suppose that $b_{t} \neq 0$. Then we can write $u$ in the form

$$
u=\hat{u}_{t} \tilde{\tilde{u}}_{t}+\sum_{\ell \in \mathfrak{L} \backslash\{t\}} \hat{u}_{\ell} \tilde{\tilde{u}}_{\ell}+\sum_{k \in K} \hat{u}_{k} u_{k}^{(4)},
$$

where $\hat{u}_{m} \in U\left(\mathfrak{G}_{-}^{f} \oplus \tilde{\mathfrak{G}}_{-} \oplus \mathfrak{G}_{-}^{\prime}\right), \tilde{\tilde{u}}_{\ell} \neq u_{k}^{(4)}$ for all $\ell, k$ and $u_{m}^{(4)} \neq u_{n}^{(4)}$ if $m \neq n$. Then $\ell(u)=|\mathfrak{L}|+|K|$. Consider the element

$$
\zeta=u-\frac{1}{b_{t}} \hat{u}_{t}\left(\sum_{\ell} b_{\ell} \tilde{\tilde{u}}_{\ell}\right)=\sum_{\ell \in \mathcal{L} \backslash\{t\}}\left(\hat{u}_{\ell}-\frac{b_{\ell}}{b_{t}} \hat{u}_{t}\right) \tilde{\tilde{u}}_{\ell}+\sum_{k \in K} \hat{u}_{k} u_{k}^{(4)} .
$$

Then $\zeta v_{\lambda}=v-\frac{1}{b_{t}} \hat{u}_{t} y_{2} y_{1} v \in N$. If $\zeta v_{\lambda}=0$ we obtain that $K=\emptyset, \hat{u}_{\ell}=\frac{b_{\ell}}{b_{t}} \hat{u}_{t}$ for all $\ell \in \mathfrak{L}, u=\hat{u}_{t} \tilde{\tilde{u}}_{t}+\sum_{\ell \in \mathfrak{L} \backslash\{t\}} \frac{b_{\ell}}{b_{t}} \hat{u}_{t} \tilde{\tilde{u}}_{\ell}=\frac{1}{b_{t}} \hat{u}_{t}\left(\sum_{\ell \in \mathfrak{L}} b_{\ell} \tilde{\tilde{u}}_{\ell}\right)$, and $v=u v_{\lambda} \in U(\mathfrak{G})[N]$. Lemma 5.1 is proved.

Suppose now that $\zeta v_{\lambda} \neq 0$. Since $\ell(\zeta)=|K|+|\mathfrak{L} \backslash\{t\}|=\ell(u)-1$, we can apply induction on $\ell(u)$ and conclude that $\tilde{\tilde{u}}_{\ell} v_{\lambda} \in[N], \ell \in \mathfrak{L} \backslash\{t\}$ and $u_{k}^{(4)} v_{\lambda} \in[N]$, 
$k \in K$. Hence $u_{k}^{(4)} v_{\lambda} \in[N]$ for all $k \in K_{0}$. It implies that $u v_{\lambda} \in U(\mathfrak{G})[N]$ and $N=U(\mathfrak{G})[N]$. This completes the proof of the lemma.

Let $N \subset M_{X}(\lambda)$. It follows from Lemma 5.1 that $N$ has a local composition series with all irreducible quotients isomorphic to $\tilde{M}(\lambda-m \delta), m \in \mathbb{Z}_{+}$. Moreover the number $[N: \tilde{M}(\lambda-m \delta)]$ does not depend on the choice of a local composition series for any $m \in \mathbb{Z}_{+}$. The following statement is a generalization of Proposition 3.4.

Proposition 5.2. Suppose $\lambda \in \mathfrak{H}^{*}, \lambda(c)=0, m \in \mathbb{Z}_{+}$and $\tilde{M}(\lambda)$ is irreducible. If $N \subset M_{X}(\lambda)$ and $\mu \in \mathfrak{H}^{*}$, then

1. $\tilde{M}(\lambda-m \delta)$ is an irreducible $\mathfrak{G}$-module.

2. $[N: \tilde{M}(\lambda-m \delta)]=\operatorname{dim}\left(\bar{M} \cap N_{\lambda-m \delta}\right)$.

3. $\operatorname{Hom}_{\mathfrak{G}}\left(M_{X}(\mu), M_{X}(\lambda)\right) \neq 0$ if and only if $\mu=\lambda-n \delta$ for some $n \geq 0$ and $\operatorname{dim} \operatorname{Hom}_{\mathfrak{G}}\left(M_{X}(\lambda-n \delta), M_{X}(\lambda)\right)=\operatorname{dim}\left(\bar{M} \cap M_{X}(\lambda)_{\lambda-n \delta}\right)$.

Proof. Statement 1 follows from Theorem 4.8, while 2 and 3 follow from Proposition 5.2.

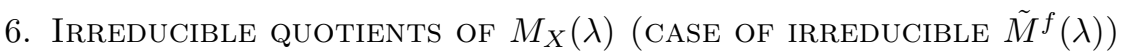

Let $\lambda \in \mathfrak{H}^{*}, \lambda(c)=0$. By Theorem 4.8, if $\tilde{M}^{f}(\lambda)$ is an irreducible $\mathfrak{G}^{f}$-module and $(\lambda, \alpha) \neq 0$ for all $\alpha \in \tilde{\pi}$, then $L_{X}(\lambda) \simeq \tilde{M}(\lambda) \simeq U\left(\mathfrak{G}_{-}^{\prime}\right) \otimes_{\mathbb{C}} U\left(\tilde{\mathfrak{G}}_{-}\right) \otimes_{\mathbb{C}} \tilde{M}^{f}(\lambda)$ as vector spaces. In this section we consider the case when $\tilde{M}^{f}(\lambda)$ is an irreducible $\mathfrak{G}^{f}$-module and there exists at least one $\alpha \in \tilde{\pi}$ such that $(\lambda, \alpha)=0$, which implies that $\tilde{M}(\lambda)$ is no longer irreducible.

Set $\mathfrak{A}=\mathfrak{G}_{-}(\tilde{\pi}(\lambda))$, where

$$
\tilde{\pi}(\lambda)=\{\alpha \in \tilde{\pi} \mid(\lambda, \alpha)=0\}, \hat{U}=U\left(\mathfrak{G}_{-}^{\prime}\right) \otimes\left(U\left(\tilde{\mathfrak{G}}_{-}\right) / U\left(\tilde{\mathfrak{G}}_{-}\right) \mathfrak{A}\right), \mathfrak{B}=U(\mathfrak{G}) \mathfrak{A} \tilde{v}_{\lambda}
$$

and $\hat{M}(\lambda)=\tilde{M}(\lambda) / \mathfrak{B}$. Let $\hat{\nu}: U(\mathfrak{G}) \rightarrow \hat{U} \otimes_{\mathbb{C}} U\left(\mathfrak{G}_{-}^{f}\right)$ and $\hat{\tau}: \tilde{M}(\lambda) \rightarrow \hat{M}(\lambda)$ be natural maps and $\hat{v}_{\lambda}=\hat{\tau}\left(\tilde{v}_{\lambda}\right)$. We will identify the elements of $\hat{U}$ with their representatives in $U\left(\mathfrak{G}_{-}^{\prime}\right) \otimes U\left(\tilde{\mathfrak{G}}_{-}\right)$.

Theorem 6.1. $L_{X}(\lambda) \simeq \hat{U} \otimes_{\mathbb{C}} \tilde{M}^{f}(\lambda)$ as vector spaces.

Proof. One can easily see that $\mathfrak{B}$ is a proper submodule of $\tilde{M}(\lambda)$. But $\mathfrak{B} \simeq \hat{U} \otimes_{\mathbb{C}}$ $U(\mathfrak{A}) \mathfrak{A} \otimes_{\mathbb{C}} \tilde{M}^{f}(\lambda)$, and thus $\hat{M}(\lambda) \simeq \hat{U} \otimes_{\mathbb{C}} \tilde{M}^{f}(\lambda)$ as vector spaces. We will show that the $\mathfrak{G}$-module $\hat{M}(\lambda)$ is irreducible. Let $N \subset \hat{M}(\lambda)$ and $0 \neq v \in N, v=u \hat{v}_{\lambda}$, where $u \in U\left(\mathfrak{G}_{-}^{\prime}\right) \otimes U\left(\mathfrak{G}_{-}^{f}\right) \otimes\left(U\left(\tilde{\mathfrak{G}}_{-}\right) / U\left(\tilde{\mathfrak{G}}_{-}\right) \mathfrak{A}\right)$. If $h t^{\prime}(u) \neq 0$, it follows from the proof of Lemma 4.4 that there exists $y \in U(\mathfrak{G})$ such that $0 \neq \hat{\nu}(y u) \in U\left(\mathfrak{G}_{-}^{f}\right) \otimes_{\mathbb{C}}$ $\left(U\left(\tilde{\mathfrak{G}}_{-}\right) / U\left(\tilde{\mathfrak{G}}_{-}\right) \mathfrak{A}\right)$.

Thus we can assume that $h t^{\prime}(u)=0$. If $\tilde{h t}(u) \neq 0$, then following the proof of Proposition 4.7 we find an element $y_{1} \in U(\mathfrak{G})$ such that $0 \neq \hat{\nu}\left(y_{1} u\right) \in U\left(\mathfrak{G}_{-}^{f}\right)$. Hence we may assume that $u \in U\left(\mathfrak{G}_{-}^{f}\right)$ and $v=u \hat{v}_{\lambda} \in \tilde{M}^{f}(\lambda)$. But $\tilde{M}^{f}(\lambda)$ is an irreducible $\mathfrak{G}^{f}$-module, which implies that $N=\hat{M}(\lambda)$. The theorem is proved.

Remark 6.2. Let $\alpha \in \tilde{\pi}$ and $(\lambda, \alpha)=0$. The structure of the module $M_{X}(\lambda)$ in this case is quite mysterious. For example, $M_{X}(\lambda)$ has irreducible subquotients which are not of type $L_{X}(\mu)$ [7]. 


\section{Irreducible Quotients of $M_{X}(\lambda)$ (CASE of Reducible $\tilde{M}^{f}(\lambda)$ )}

In this section we will assume that the $\mathfrak{G}^{f}$-module $\tilde{M}^{f}(\lambda)$ is reducible. Thus $L_{X}(\lambda)$ is the unique irreducible quotient of $\hat{M}(\lambda)$ and $L^{f}(\lambda)$ is the unique irreducible quotient of $\tilde{M}^{f}(\lambda)$.

Remark 7.1. One would expect that $L_{X}(\lambda) \simeq \hat{U} \otimes L_{X}^{f}(\lambda)$, but this is not always the case. For example, if $L_{X}^{f}(\lambda)=\mathbb{C} v$ and $(\lambda, \beta)=0$ for some $\beta \in \pi^{\prime}$, then $U(\mathfrak{G})\left(\mathfrak{G}_{-\beta} \otimes v\right)$ is a proper submodule of $\hat{U} \otimes \mathbb{C} v$ and thus $L_{X}(\lambda) \not \hat{U} \otimes \mathbb{C} v$.

Conjecture 7.2. If $\lambda \in \mathfrak{H}^{*}, \lambda(c)=0$ and $\operatorname{dim} L_{X}^{f}(\lambda)>1$, then $L_{X}(\lambda) \simeq \hat{U} \otimes$ $L_{X}^{f}(\lambda)$.

We will prove this conjecture for $\lambda$ in "general position".

Definition 7.3. Let $\lambda \in \mathfrak{H}^{*}, \lambda(c)=0$. We will say that $\lambda$ is in general position if $(\mu, \beta) \neq 0$ for all $\mu \in \lambda+Q_{\pi^{f}}$ and any $\beta \in \pi^{\prime} \backslash \tilde{\pi}$.

Example 7.4. Any $\lambda \in \mathfrak{H}^{*}$ such that $\lambda(c)=0$ and $\frac{2(\lambda, \beta)}{(\beta, \beta)} \notin \mathbb{Z}$ for all $\beta \in \pi^{\prime} \backslash \tilde{\pi}$ is in general position.

Let $u \in \hat{U} \otimes U\left(\mathfrak{G}_{-}^{f}\right)$ be a homogeneous element. We will say that $u$ is represented in a normal form if $u=\sum_{k \in K} u_{k} u_{k}^{f}$, where $u_{k} \in \hat{U}, u_{k}^{f} \in U\left(\mathfrak{G}_{-}^{f}\right), u_{k}$ are linearly independent and $u_{i}^{f} \notin \mathbb{C}^{*} u_{j}^{f}$ if $i \neq j$. Obviously, if $|K|>1$ then $u$ has many different normal forms.

Using the PBW Theorem we can choose a basis in $U\left(\mathfrak{G}_{-}^{f}\right)$ consisting of the monomials $u^{(\delta)} u^{(f)}$ where $u^{(\delta)} \in U\left(G_{-}\right) \cap U\left(\mathfrak{G}_{-}^{f}\right)$ and $u^{(f)}$ contains no elements of $U\left(G_{-}\right)$. Thus any element $u \in U\left(\mathfrak{G}_{-}^{f}\right)$ can be written uniquely as a linear combination of certain monomials $u_{i}^{(\delta)} u_{i}^{(f)}$. Suppose that $u \in U\left(\mathfrak{G}_{-}^{f}\right)_{\eta+m \delta}, u_{i}^{(f)} \in$ $U\left(\mathfrak{G}_{-}^{f}\right)_{\eta_{i}+m_{i} \delta}$ and $\eta, \eta_{i} \in Q, m, m_{i} \in \mathbb{Z}$ for all $i$. Then we set $d(u)=\max _{i}\left|m_{i}\right|$ and $\bar{d}(u)=|m|-d(u)$. With each homogeneous element $u \in \hat{U} \otimes U\left(\mathfrak{G}_{-}^{f}\right)$ represented in a normal form $u=\sum_{k \in K} u_{k} u_{k}^{f}$ we will associate $D(u)=\max _{k \in K} d\left(u_{k}^{f}\right)$ and the set $S(u)=\left\{k \in K \mid d\left(u_{k}^{f}\right)=D(u)\right\}$. Note that $D(u)$ is independent of the choice of a normal form and depends only of $u$. On the other hand the set $S(u)$ is determined by a given normal form.

Let $\mathfrak{H}^{\prime}=\mathfrak{H} \cap \mathfrak{G}\left(\pi^{\prime}\right), G^{\prime}=G \cap \mathfrak{G}\left(\pi^{\prime}\right)$. Consider the following decomposition of $U\left(\mathfrak{G}\left(\pi^{\prime}\right)\right): U\left(\mathfrak{G}\left(\pi^{\prime}\right)\right)=U\left(\mathfrak{H}^{\prime} \oplus G^{\prime}\right) \oplus\left(U\left(\mathfrak{G}\left(\pi^{\prime}\right)\right) \mathfrak{G}_{+}\left(\pi^{\prime}\right)+\mathfrak{G}_{-}\left(\pi^{\prime}\right) U\left(\mathfrak{G}\left(\pi^{\prime}\right)\right)\right)$, and the corresponding projection $\nu_{0}: U\left(\mathfrak{G}\left(\pi^{\prime}\right)\right) \rightarrow U\left(\mathfrak{H}^{\prime} \oplus G^{\prime}\right)$.

Proposition 7.5. Let $\lambda, \mu \in \mathfrak{H}^{*}, \lambda(c)=0$ and $\lambda$ is in general position, $N \subset \hat{M}(\lambda)$, $0 \neq u \hat{v}_{\lambda} \in N_{\mu}$ and $u=\sum_{k \in K} u_{k} u_{k}^{f}$ is a normal form of $u$ such that $h t^{f}\left(u_{k}\right)=0$ for all $k \in K$. Then $u_{k}^{f} \hat{v}_{\lambda} \in N$ for all $k \in K$.

Proof. Denote $v=u \hat{v}_{\lambda}$. Assume that $u_{k} \in \hat{U}_{\eta_{k}}$ and set $\mu_{k}=\mu-\eta_{k}, \eta_{k} \in Q, k \in K$. Then $\left(\mu_{k}, \beta\right) \neq 0$ for any $\beta \in \pi^{\prime}$ and $k \in K$, since $\lambda$ is in "general position". We will prove the statement by induction on $|K|, D(u)$ and $|S(u)|$ simultaneously.

Step 1. Let $|K|=1$. Then $u=u^{\prime} u^{f}$, where $u^{\prime}$ is a homogeneous element of $\hat{U}$ and $h t^{f}\left(u^{\prime}\right)=0$. Consider a $U\left(\mathfrak{G}_{-}\left(\pi^{\prime}\right)\right)$-module $V=U\left(\mathfrak{G}_{-}\left(\pi^{\prime}\right)\right) u^{f} \hat{v}_{\lambda}$ with $\mathfrak{G}_{+}\left(\pi^{\prime}\right) u^{f} \hat{v}_{\lambda}=0$ and $\mathfrak{G}_{-\beta}\left(u^{f} \hat{v}_{\lambda}\right)=u^{f} \mathfrak{G}_{-\beta} \hat{v}_{\lambda}=0$ for all $\beta \in \tilde{\pi}(\mu)$. Obviously, $v \in N \cap V$, and it follows from Proposition 3.2 that $u^{f} \hat{v}_{\lambda} \in U\left(\mathfrak{G}\left(\pi^{\prime}\right)\right) v \subset N$. 
Step 2. Suppose now that $|K|>1$ and $D(u)=0$. Consider $\tilde{K}=\{k \in$ $K \mid \bar{d}\left(u_{k}^{f}\right) \leq \bar{d}\left(u_{j}^{k}\right)$ for all $\left.j \in K\right\}$. Let $k_{0} \in \tilde{K}$. Then by Step 1 there exists $y \in U\left(\mathfrak{G}\left(\pi^{\prime}\right)\right)$ for which $y u_{k_{0}} u_{k_{0}}^{f} \hat{v}_{\lambda}=a_{k_{0}} u_{k_{0}}^{f} \hat{v}_{\lambda}, a_{k_{0}} \in \mathbb{C}^{*}$. Since $u$ is homogeneous and $h t^{f}\left(u_{k}\right)=0$ for all $k$, we have $\nu_{0}\left(y u_{k}\right) \in U(\mathfrak{H}) \otimes U(G)_{0}, k \in$ $\tilde{K} \backslash\left\{k_{0}\right\}$ and $\nu_{0}\left(y u_{k}\right) \in U(\mathfrak{H}) \otimes_{\mathbb{C}} U(G)_{0} G_{+}, k \in K \backslash \tilde{K}$. As $d\left(u_{k}^{f}\right)=0$ for all $k \in K$, we conclude that $y u_{k} u_{k}^{f} \hat{v}_{\lambda}=a_{k} u_{k}^{f} \hat{v}_{\lambda}, a_{k} \in \mathbb{C}, k \in \tilde{K} \backslash\left\{k_{0}\right\}$, and $y u_{k} u_{k}^{f} \hat{v}_{\lambda}=0, k \in K \backslash \tilde{K}$. Hence $y v=\sum_{k \in K} y u_{k} u_{k}^{f} \hat{v}_{\lambda}=\sum_{k \in K} \nu_{0}\left(y u_{k}\right) u_{k}^{f} \hat{v}_{\lambda}=$ $\sum_{k \in \tilde{K}} a_{k} u_{k}^{f} \hat{v}_{\lambda}$. Suppose that $\sum_{k \in \tilde{K}} a_{k} u_{k}^{f}=0$. Then $u_{k_{0}}^{f}=\sum_{k \in K \backslash\left\{k_{0}\right\}} \frac{a_{k}}{a_{k_{0}}} u_{k}^{f}$ and $u=\sum_{k \in K \backslash\left\{k_{0}\right\}} u_{k} u_{k}^{f}+u_{k_{0}} u_{k_{0}}^{f}=\sum_{k \in \tilde{K} \backslash\left\{k_{0}\right\}}\left(u_{k}+\frac{a_{k}}{a_{k_{0}}} u_{k_{0}}\right) u_{k}^{f}+\sum_{k \in K \backslash \tilde{K}} u_{k} u_{k}^{f}$. Applying induction on $|K|$, we conclude that $u_{k}^{f} \hat{v}_{\lambda} \in N$ for all $k \in K \backslash\left\{k_{0}\right\}$, and thus $u_{k}^{f} \hat{v}_{\lambda} \in N$ for all $k \in K$. Suppose now that $\sum_{k \in \tilde{K}} a_{k} u_{k}^{f} \neq 0$. Then $y v \neq 0$ and $0 \neq a_{k_{0}} v-u_{k_{0}} y v=\left(a_{k_{0}} u-u_{k_{0}} y u\right) \hat{v}_{\lambda}=u^{\prime} \hat{v}_{\lambda} \in N$, where $u^{\prime}=$ $\sum_{k \in \tilde{K} \backslash\left\{k_{0}\right\}}\left(a_{k_{0}} u_{k}-a_{k} u_{k_{0}}\right) u_{k}^{f}+\sum_{k \in K \backslash \tilde{K}} a_{k_{0}} u_{k} u_{k}^{f}$ is represented in a normal form. The induction on $|K|$ implies that $u_{k}^{f} \hat{v}_{\lambda} \in N$ for all $k \in K \backslash\left\{k_{0}\right\}$, and therefore $u_{k}^{f} \hat{v}_{\lambda} \in N$ for all $k \in K$.

Step 3. Now let $|K|>1, D(u)>0$ and $|S(u)|=1$. Suppose that $S(u)=$ $\left\{k_{0}\right\}$ and consider an element $y \in U\left(\mathfrak{G}\left(\pi^{\prime}\right)\right)$ as in Step 2. Let $y u_{k_{0}} u_{k_{0}}^{f} \hat{v}_{\lambda}=$ $a_{k_{0}} u_{k_{0}}^{f} \hat{v}_{\lambda}, a_{k_{0}} \in \mathbb{C}^{*}$. Since $|S(u)|=1$, we obtain $y v=y u \hat{v}_{\lambda}=a_{k_{0}} u_{k_{0}}^{f} \hat{v}_{\lambda}+$ $\sum_{k \in K \backslash\left\{k_{0}\right\}} \nu_{0}\left(y u_{k}\right) u_{k}^{f} \hat{v}_{\lambda} \neq 0$ and $N \ni a_{k_{0}} v-u_{k_{0}} y v=u^{\prime} \hat{v}_{\lambda}$, where

$$
u^{\prime}=\sum_{k \in K \backslash\left\{k_{0}\right\}} a_{k_{0}} u_{k} u_{k}^{f}-\sum_{k \in K \backslash\left\{k_{0}\right\}} u_{k_{0}} \nu_{0}\left(y u_{k}\right) u_{k}^{f} .
$$

If $\eta_{k}=\eta_{k_{0}}$ then $\nu_{0}\left(y u_{k}\right) \in S(\mathfrak{H}) \otimes U(G)_{0}$; otherwise

$$
\nu\left(y u_{k}\right) \in S(\mathfrak{H}) \otimes\left(U(G)\left(G_{-} \oplus G_{+}\right)\right) .
$$

Since the $u_{k}$ are linearly independent, this implies that $0 \neq u^{\prime} \hat{v}_{\lambda} \in N$ and $D\left(u^{\prime}\right)<$ $D(u)$. Rewriting $u^{\prime}$ in a normal form if necessary, we can apply induction on $D(u)$. Hence $u_{k}^{f} \hat{v}_{\lambda} \in N$ for all $k \in K \backslash\left\{k_{0}\right\}$, implying $u_{k}^{f} \hat{v}_{\lambda} \in N$ for all $k \in K$ by induction on $|K|$.

Step 4. Suppose now that $|K|>1, D(u)>0$ and $|S(u)|>1$. Denote $\tilde{K}=$ $\left\{k \in S(u) \mid \bar{d}\left(u_{k}^{f}\right) \leq \bar{d}\left(u_{j}^{f}\right)\right.$ for all $\left.j \in S(u)\right\}$. Let $k_{0} \in \tilde{K}$. Note that $\mu_{k}=\mu_{k_{0}}$ for all $k \in \tilde{K}$. As in Step 2, consider an element $y \in U\left(\mathfrak{G}\left(\pi^{\prime}\right)\right)$ for which $\nu_{0}\left(y u_{k_{0}}\right) \in$ $S(\mathfrak{H}) \otimes U(G)_{0}$ and $y u_{k_{0}} u_{k_{0}}^{f} \hat{v}_{\lambda}=a_{k_{0}} u_{k_{0}}^{f} \hat{v}_{\lambda}, a_{k_{0}} \in \mathbb{C}^{*}$. Then $N \ni a_{k_{0}} v-u_{k_{0}} y v=$ $\left(\sum_{k \in K \backslash\left\{k_{0}\right\}} a_{k_{0}} u_{k} u_{k}^{f}-\sum_{k \in K \backslash\left\{k_{0}\right\}} u_{k_{0}} \nu_{0}\left(y u_{k}\right) u_{k}^{f}\right) \hat{v}_{\lambda}, \nu_{0}\left(y u_{k}\right) \in S(\mathfrak{H}) \otimes U(G)_{0}$ if $\mu_{k}=\mu_{k_{0}}$, and $\nu_{0}\left(y u_{k}\right) \in S(\mathfrak{H}) \otimes\left(U(G)\left(G_{+} \oplus G_{-}\right)\right)$otherwise. Clearly, $\hat{\nu}\left(y u_{k} u_{k}^{f}\right)=$ $a_{k} u_{k}^{f}+u_{k}^{\prime}, a_{k} \in \mathbb{C}, u_{k}^{\prime} \in U\left(\mathfrak{G}_{-}^{f}\right)$ for $k \in \tilde{K} \backslash\left\{k_{0}\right\}$, and $\hat{\nu}\left(y u_{k} u_{k}^{f}\right)=u_{k}^{\prime} \in U\left(\mathfrak{G}_{-}^{f}\right)$ for $k \in K \backslash \tilde{K}$, where $d\left(u_{k}^{\prime}\right)<D(u)$ for all $k \in K \backslash\left\{k_{0}\right\}$. Set $u^{\prime \prime}=\sum_{k \in K \backslash\left\{k_{0}\right\}} u_{k}^{\prime}$, $d\left(u^{\prime \prime}\right)<D(u)$. Thus $a_{k_{0}} v-u_{k_{0}} y v=u^{\prime} \hat{v}_{\lambda} \neq 0$, where

$$
u^{\prime}=\sum_{k \in \tilde{K} \backslash\left\{k_{0}\right\}}\left(a_{k_{0}} u_{k}-a_{k} u_{k_{0}}\right) u_{k}^{f}+\sum_{k \in K \backslash \tilde{K}} a_{k_{0}} u_{k} u_{k}^{f}-u_{k_{0}} u^{\prime \prime} .
$$

If $u^{\prime \prime} \notin \mathbb{C}^{*} u_{k}^{f}$ for all $k \in K \backslash \tilde{K}$, then $u^{\prime}$ is represented in a normal form, $\left|S\left(u^{\prime}\right)\right|<$ $|S(u)|$ and induction on $|S(u)|$ implies $u_{k}^{f} \hat{v}_{\lambda} \in N$ for all $k \in K \backslash\left\{k_{0}\right\}$. By induction on $|K|$ we conclude that $u_{k}^{f} \hat{v}_{\lambda} \in N$ for all $k \in K$. Suppose now that $u^{\prime \prime}=a u_{j}^{f}$ 
for some $j \in K \backslash \tilde{K}$ and $a \in \mathbb{C}^{*}$. Then $u^{\prime}=\sum_{k \in \tilde{K} \backslash\left\{k_{0}\right\}}\left(a_{k_{0}} u_{k}-a_{k} u_{k_{0}}\right) u_{k}^{f}+$ $\sum_{\substack{k \in K \backslash \tilde{K} \\ k \neq j}} a_{k_{0}} u_{k} u_{k}^{f}+\left(a_{k_{0}} u_{j}-a u_{k_{0}}\right) u_{j}^{f}$ is the normal form of $u^{\prime}$ and $\left|S\left(u^{\prime}\right)\right|<|S(u)|$.

By induction on $|S(u)|$ and $|K|$ we conclude that $u_{k}^{f} \hat{v}_{\lambda} \in N$ for all $k \in K$, which completes the proof.

Let $u \in \hat{U} \otimes U\left(\mathfrak{G}_{-}^{f}\right)$ be a homogeneous element. We will say that $u$ is represented in the reduced normal form $u=\sum_{i} u_{i} u_{i}^{f}$ if $u_{i}=\sum_{j} a_{i j} \bar{u}_{i j}$ for each $i$, where $a_{i j} \in \mathbb{C}^{*}, \bar{u}_{i j}$ are monomials in $\hat{u}$ and $\bar{u}_{i j}=\bar{u}_{k \ell}$ only if $i=k, j=\ell$. Obviously, any non-zero homogeneous element of $\hat{U} \otimes U\left(\mathfrak{G}_{-}^{f}\right)$ has a reduced normal form.

Lemma 7.6. Let $u=\sum_{i} u_{i} u_{i}^{f}$ be the reduced normal form of $u \in \hat{U} \otimes U\left(\mathfrak{G}_{-}^{f}\right)$, where $h t^{f}\left(u_{i}\right) \leq d$ for all $i$. Then there exists $y \in \mathfrak{G}_{+}^{\prime}$ such that $\hat{u}=\hat{\nu}(y u) \neq 0$ and $\hat{u}$ has the reduced normal form $\hat{u}=\sum_{j} \hat{u}_{j} \hat{u}_{j}^{f}$, where $\hat{u}_{j}^{f}=u_{i}^{f}$ for at least one pair of indexes $(i, j)$ and $h t^{f}\left(\hat{u}_{j}\right) \leq d-1$ for all $j$.

Proof. Let $J=\left\{i \mid h t^{f}\left(u_{i}\right)=d\right\}$. For simplicity we may assume that all $u_{i}$ are monomials in $\hat{U}$ and $u_{i}=X_{-\phi_{1 i}+n_{1 i} \delta} \ldots X_{-\phi_{s(i) i}+n_{s(i) i} \delta} u_{i}^{\prime}$, where $h t^{\prime}\left(\phi_{j i}\right) \neq 0$ for all $i, j, \sum_{j} h t^{f}\left(\phi_{j i}\right) \leq d, 0<h t^{f}\left(\phi_{s(i) i} \leq \ldots \leq h t^{f}\left(\phi_{2 i}\right) \leq h t^{f}\left(\phi_{1 i}\right)\right.$ and $h t^{f}\left(u_{i}^{\prime}\right)=0$ for all $i$. We can also assume that $u_{i}$ are numerated in such a way that $1 \in J, h t^{f}\left(\phi_{s(1) 1}\right) \leq h t^{f}\left(\phi_{s(i) i}\right)$ for any $i \in J$, if $h t^{f}\left(\phi_{s(1) 1}\right)=h t^{f}\left(\phi_{s(i) i}\right)$ then $h t^{\prime}\left(\phi_{s(1) 1}\right) \leq h t^{\prime}\left(\phi_{s(i) i}\right)$, and $-\phi_{s(1) 1}+n_{s(1) 1} \delta=-\phi_{k i}+n_{k i} \delta$ implies $k=$ $s(i)$. Suppose that $\phi_{s(1) 1} \in \Delta$. By Lemma 3.1, (5), there exists $\psi \in \Delta_{+}^{\prime}$ such that $h t^{f}(\psi)=1$ and $\psi-\phi_{s(1) 1} \in \Delta_{-}^{\prime}$. Then for large enough $m \in \mathbb{Z}_{+}$and for $0 \neq y \in \mathfrak{G}_{\psi+m \delta}$ we have that $\left[y, u_{i}^{\prime}\right]=0, \hat{\nu}\left(y u_{i}^{f}\right)=0,\left[y, u_{i}\right] \in \hat{U}, h t^{f}\left(\left[y, u_{i}\right]\right) \leq$ $d-1$ for $i \in J$ and $h t^{f}\left(\left[y, u_{j}\right]\right) \leq d-2$ for $j \notin J$. Also note that a monomial $x=X_{-\phi_{11}+n_{11} \delta} \ldots X_{-\phi_{s(1)-11}+n_{s(1)-11} \delta}\left[X_{\psi+m \delta}, X_{-\phi_{s(1) 1}+n_{s(1) 1} \delta}\right] u_{1}^{\prime} \neq 0$, and it will appear in $\left[y, u_{i}\right]$ only for $i=1$. Hence, $\hat{u}=\hat{\nu}(y u)=\hat{\nu}([y, u])=\sum_{i}\left[y, u_{i}\right] u_{i}^{f} \neq 0$, and if $\hat{u}=\sum_{j} \hat{u}_{j} \hat{u}_{j}^{f}$ is the reduced normal form then $\hat{u}_{k}$ contains $x$ for some $k$ and $\hat{u}_{k}^{f}=u_{1}^{f}$. If $\phi_{s(1) 1} \notin \Delta$ then $\frac{1}{2} \phi_{s(1) 1} \in \Delta, \hat{\nu}\left(X_{\frac{1}{2} \phi_{s(1) 1}} u\right) \neq 0$, and the same arguments as above complete the proof.

If $N$ is a $\mathfrak{G}$-submodule of $\hat{M}(\lambda)$, we set $N^{f}=\sum_{\mu \in \mathfrak{H}^{*}} N_{\mu}, 0<\operatorname{dim} N_{\mu}<\infty$. Clearly, $N^{f}$ is isomorphic to a $\mathfrak{G}^{f}$-submodule of $\tilde{M}^{f}(\lambda)$.

Theorem 7.7. Let $\lambda \in \mathfrak{H}^{*}, \lambda(c)=0$ and $\lambda$ is in general position.

1. If $N \subset \hat{M}(\lambda)$ then $N \simeq \hat{U} \otimes_{\mathbb{C}} N^{f}$ as vector spaces.

2. $L_{X}(\lambda) \simeq \hat{U} \otimes_{\mathbb{C}} L^{f}(\lambda)$ as vector spaces.

Proof. Let $0 \neq v \in N$. Without loss of generality we can assume that $v$ is a weight vector. Then $v=u \hat{v}_{\lambda}$ for some homogeneous element $u \in \hat{U} \otimes U\left(\mathfrak{G}_{-}^{f}\right)$. Let $u=\sum_{i} u_{i} u_{i}^{f}$ be the reduced normal form of $u$ and $d=\max _{i} h t^{f}\left(u_{i}\right)$. Using Lemma 7.6 we construct a sequence $y_{1}, \ldots, y_{d} \in \mathfrak{G}_{+}^{\prime}$ such that $\hat{u}_{j}=\hat{\nu}\left(y_{j} \ldots y_{1} u\right)=$ $\sum_{i}\left[y_{j} \ldots\left[y_{1}, u_{i}\right] \ldots\right] u_{i}^{f} \neq 0,\left[y_{j} \ldots\left[y_{1}, u_{i}\right] \ldots\right] \in \hat{U}, h t^{f}\left(\left[y_{j} \ldots\left[y_{1}, u_{i}\right] \ldots\right]\right) \leq d-j$, $j=1, \ldots, d$, and for each $j$, if $\hat{u}_{j}=\sum_{k \in K_{j}} \hat{u}_{j k} \hat{u}_{j k}^{f}$ is the reduced normal form of $\hat{u}_{j}$, then one can find a pair $(k, \ell)$ for which $\hat{u}_{j k}^{f}=\hat{u}_{j-1 \ell}^{f}$. Since $y_{d} \ldots y_{1} v=\hat{u}_{d} v \in N$ and $h t^{f}\left(\hat{u}_{d k}\right)=0$, we can apply Proposition 7.5 and conclude that $\hat{u}_{d k}^{f} \hat{v}_{\lambda} \in N$ for all $k \in K_{d}$. In particular, $\hat{u}_{d-1 \ell}^{f} \hat{v}_{\lambda} \in N$ for some $\ell \in K_{d-1}$. Thus $\hat{u}_{d-1} \hat{v}_{\lambda}-$ 
$\hat{u}_{d-1 \ell} \hat{u}_{d-1 \ell}^{f} \hat{v}_{\lambda}=\sum_{k \in K_{d-1} \backslash\{\ell\}} \hat{u}_{d-1 k} \hat{u}_{d-1 k}^{f} \hat{v}_{\lambda} \in N$ and $\hat{u}_{d-1 k}^{f} \hat{v}_{\lambda} \in N$ for all $k \in K_{d-1}$ by induction on $\left|K_{d-1}\right|$. We conclude by induction on $d$ that $u_{i}^{f} \hat{v}_{\lambda} \in N$ for all $i$, which completes the proof of $(1)$. Since $\hat{M}(\lambda) \simeq \hat{U} \otimes_{\mathbb{C}} \tilde{M}^{f}(\lambda)$ and $L_{X}(\lambda)$ is the unique irreducible quotient of $\hat{M}(\lambda)$, the statement (2) follows immediately from (1).

Set $\mathfrak{A}=\mathfrak{G}_{-}(\tilde{\pi}(\lambda))$.

Corollary 7.8. Let $\lambda \in \mathfrak{H}^{*}, \lambda(c)=0$ and assume $\lambda$ is in general position.

1. If $N \subset \hat{M}(\lambda)$ then $N \simeq(U(\mathfrak{G}) / U(\mathfrak{G}) \mathfrak{A}) \otimes_{U\left(\mathbf{p}_{X}\right)} N^{f}$, where $u_{X}^{+}$acts trivially on $N^{f}$.

2. $L_{X}(\lambda) \simeq(U(\mathfrak{G}) / U(\mathfrak{G}) \mathfrak{A}) \otimes_{U\left(\mathbf{p}_{X}\right)} L^{f}(\lambda)$, where $\mathbf{u}_{X}^{+}$acts trivially on $L^{f}(\lambda)$.

Proof. Since $\mathbf{u}_{X}^{+} N^{f}=\mathbf{u}_{X}^{+} L^{f}(\lambda)=\mathfrak{A} N^{f}=\mathfrak{A} L^{f}(\lambda)=0$, the statements follow from Theorem 7.7.

Denote $\check{M}(\lambda)=M_{X}(\lambda) / U(\mathfrak{G}) \mathfrak{A} v_{\lambda}$. We have a chain of epimorphisms: $M_{X}(\lambda) \rightarrow$ $\check{M}(\lambda) \rightarrow \hat{M}(\lambda) \rightarrow L_{X}(\lambda)$. Thus $L_{X}(\lambda)$ is the unique irreducible quotient of $\check{M}(\lambda)$ and $\check{M}^{f}(\lambda) \simeq M^{f}(\lambda)$.

Corollary 7.9. Let $\lambda \in \mathfrak{H}^{*}, \lambda(c)=0$ and $\lambda$ is in general position. If $N \subset \check{M}(\lambda)$ then $N \simeq(U(\mathfrak{G}) / U(\mathfrak{G}) \mathfrak{A}) \otimes_{U\left(\mathbf{p}_{X}\right)} N^{f}$, where $\mathbf{u}_{X}^{+}$acts trivially on $N^{f}$.

Proof. Follows from Corollary 7.8, (1).

Corollary 7.10. Let $\mu, \nu \in \mathfrak{H}^{*}, \mu(c)=\nu(c)=0$ and assume both $\mu$, $\nu$ are in general position.

1. $\operatorname{Hom}_{\mathfrak{G}}(\hat{M}(\mu), \hat{M}(\nu))=\operatorname{Hom}_{\tilde{\mathfrak{G}} f}\left(\tilde{M}^{f}(\mu), \tilde{M}^{f}(\nu)\right)$.

2. $\operatorname{Hom}_{\mathfrak{G}}(\check{M}(\mu), \check{M}(\nu)) \simeq \operatorname{Hom}_{\mathbf{m}}\left(M^{f}(\mu), M^{f}(\nu)\right)$.

3. Modules $\hat{M}(\mu)$ and $\check{M}(\mu)$ have local composition series and $\left[\hat{M}(\mu): L_{X}(\nu)\right]=$ $\left[\tilde{M}^{f}(\mu): L^{f}(\nu)\right],\left[\check{M}(\mu): L_{X}(\nu)\right]=\left[M^{f}(\mu): L^{f}(\nu)\right]$.

Proof. Follows from Corollaries 7.8, 7.9 and the fact that both $M^{f}(\mu)$ and $\tilde{M}^{f}(\mu)$ have local compositon series [13].

Corollary 7.11. Let $\mu, \nu \in \mathfrak{H}^{*}, \mu(c)=\nu(c)=0$ and assume both $\mu, \nu$ are in general position. Then

1. $\operatorname{Hom}_{\mathfrak{G}}(\check{M}(\mu), \check{M}(\nu)) \neq 0$ if and only if $\left[\check{M}(\nu): L_{X}(\mu)\right] \neq 0$.

2. $\operatorname{Hom}_{\mathfrak{G}}(\hat{M}(\mu), \hat{M}(\nu)) \neq 0$ if and only if $\left[\hat{M}(\nu): L_{X}(\mu)\right] \neq 0$.

Proof. Since

$$
\operatorname{Hom}_{\mathbf{m}}\left(M^{f}(\mu), M^{f}(\nu)\right) \neq 0 \Leftrightarrow\left[M^{f}(\nu): L^{f}(\mu)\right] \neq 0
$$

and

$$
\operatorname{Hom}_{\tilde{\mathfrak{G}} f}\left(\tilde{M}^{f}(\mu), \tilde{M}^{f}(\nu)\right) \neq 0 \Leftrightarrow\left[\tilde{M}^{f}(\nu): L^{f}(\mu)\right] \neq 0
$$

by [13] (Ch.2.11, Theorem 1), the statements follow from Corollary 7.10. 


\section{Strong BGG Resolution for modules $\hat{M}\left(\lambda_{0}\right)$}

In this section we assume that $X$ is connected, i.e. the corresponding CoxeterDynkin diagram is connected, and thus $\mathfrak{G}^{f}$ is the derived algebra of an affine Lie algebra. Let $\bar{\pi}^{f}$ be a basis of $\Delta^{f}$ containing $\pi^{f}$ and $\lambda_{0} \in \mathfrak{H}^{*}$ such that $\left(\lambda_{0}, \alpha\right)=0$ for all $\alpha \in \bar{\pi}^{f}$. Let $W_{X}$ be the Weyl group of $\mathfrak{G}^{f}, \ell$ be a length function and $s_{\beta}$ denote the reflection corresponding to the root $\beta$. For $w$ and $w^{\prime}$ in $W_{X}$ we write $w \leftarrow w^{\prime}$ if there exists a root $\beta \in \Delta_{+}^{f} \cap \Delta^{r e}$ such that $w=s_{\beta} w^{\prime}$ and $\ell(w)=\ell\left(w^{\prime}\right)+1$. The Bruhat order on $W_{X}$ is defined by : $w \leq w^{\prime}$ if $w=w^{\prime}$ or if there are $w_{1}, \ldots, w_{r} \in W_{X}$ such that $w=w_{1} \leftarrow \ldots \leftarrow w_{r}=w^{\prime}$.

For $w \in W_{X}$ and $\mu \in \mathfrak{H}^{*}$, define $w \circ \mu=w\left(\mu+\rho_{X}\right)-\rho_{X}$, where $\rho_{X} \in \mathfrak{H}^{*}$ is any fixed element such that $\rho_{X}(\alpha)=1$ for all $\alpha \in \bar{\pi}^{f}$.

Theorem 8.1 (cf. Theorem 5.2 in [3]). Let $X$ be connected, $\lambda_{0} \in \mathfrak{H}^{*},\left(\lambda_{0}, \alpha\right)=0$ for all $\alpha \in \bar{\pi}^{f}$ and $w, w^{\prime} \in W_{X}$. Then

$$
\begin{aligned}
\operatorname{dim} \operatorname{Hom}_{\mathfrak{G}}\left(\hat{M}\left(w^{\prime} \circ \lambda_{0}\right), \hat{M}\left(w \circ \lambda_{0}\right)\right)=1 & \Leftrightarrow w^{\prime} \leq w \\
& \Leftrightarrow\left[\hat{M}\left(w \circ \lambda_{0}\right): L_{X}\left(w^{\prime} \circ \lambda_{0}\right)\right] \neq 0 .
\end{aligned}
$$

Proof. Since $\operatorname{Hom}_{\mathfrak{G}}\left(\hat{M}\left(w^{\prime} \circ \lambda_{0}\right), \hat{M}\left(w \circ \lambda_{0}\right)\right) \simeq \operatorname{Hom}_{\tilde{\mathfrak{G}} f}\left(\tilde{M}^{f}\left(w^{\prime} \circ \lambda_{0}\right), \tilde{M}^{f}\left(w \circ \lambda_{0}\right)\right)$ by Corollary 7.10, (1) and $\left[\hat{M}\left(w \circ \lambda_{0}\right): L_{X}\left(w^{\prime} \circ \lambda_{0}\right)\right]=\left[\tilde{M}^{f}\left(w \circ \lambda_{0}\right): L^{f}\left(w^{\prime} \circ \lambda_{0}\right)\right]$ by Corollary 7.10, (3), the statement follows from [14], Theorem 8.15.

For any $i \in \mathbb{Z}_{+}$, denote $W_{X}^{(i)}=\left\{w \in W_{X} \mid \ell(w)=i\right\}$ and set

$$
C_{i}=\bigoplus_{w \in W_{X}^{(i)}} \hat{M}\left(w \circ \lambda_{0}\right)
$$

If $w, w^{\prime} \in W_{X}$ we fix $0 \neq i_{w, w^{\prime}}\left(\lambda_{0}\right) \in \operatorname{Hom}_{\mathfrak{G}}\left(\hat{M}\left(w^{\prime} \circ \lambda_{0}\right), \hat{M}\left(w \circ \lambda_{0}\right)\right)$. Let $d_{j}: C_{j} \rightarrow$ $C_{j-1}, j \geq 1$, defined by $d_{j}=\bigoplus b_{w, w^{\prime}}^{j} i_{w, w^{\prime}}\left(\lambda_{0}\right), w \in W_{X}^{(j)}, w^{\prime} \in W_{X}^{(j-1)}$, where $b_{w, w^{\prime}}^{j} \in\{ \pm 1\}$ are defined by [14], Lemma 9.6 if $w \leftarrow w^{\prime}$ and $b_{w, w^{\prime}}^{j}=0$ otherwise.

Theorem 8.2 (cf. Theorem 5.4 in [3]). Let $X$ be connected, $\lambda_{0} \in \mathfrak{H}^{*},\left(\lambda_{0}, \alpha\right)=0$ for all $\alpha \in \bar{\pi}^{f}$ and $\eta: \hat{M}\left(\lambda_{0}\right) \rightarrow L_{X}\left(\lambda_{0}\right)$ be the canonical projection. Then the sequence

$$
\ldots C_{j} \stackrel{d_{j}}{\rightarrow} C_{j-1} \rightarrow \ldots \rightarrow C_{1} \stackrel{d_{1}}{\rightarrow} \hat{M}\left(\lambda_{0}\right) \stackrel{\eta}{\rightarrow} L_{X}\left(\lambda_{0}\right) \rightarrow 0, \quad(j \geq 1)
$$

is exact.

Proof. Follows from Corollary 7.8, (1), Corollary 7.10, (1) and Theorem 9.7 in [14].

\section{Category $\mathfrak{O}_{X}(\lambda)$}

In this section, following [2], we define certain categories of $\mathfrak{G}$-modules, which contain the Verma type modules and their irreducible quotients, and show that the BGG duality holds in these categories.

Let $\lambda \in \mathfrak{H}^{*}, \lambda(c)=0$ and $\lambda$ be in general position. Consider the full subcategory $\mathfrak{O}^{f}(\lambda)$ of the category of weight $\mathbf{m}$-modules, whose objects $V$ satisfy

1. $P(V) \subset\left\{\mu \in \mathfrak{H}^{*} \mid \mu \leq \lambda\right\}$.

2. $\operatorname{dim} V_{\mu}<\infty$ for all $\mu \in P(V)$. 
The category $\mathfrak{O}^{f}(\lambda)$ is stable under the operations of taking submodules, quotients and finite direct sums. Note that for any $\mu \leq \lambda$, the modules $M^{f}(\mu)$ and $L^{f}(\mu)$ are objects of $\mathfrak{O}^{f}(\lambda)$ and, moreover, the modules $L^{f}(\mu)$ exhaust all irreducible objects in $\mathfrak{O}^{f}(\lambda)$.

Recall that $\mathbf{m}$ has a triangular decomposition and thus all the results of [14], sections 4-6, can be applied to the category $\mathfrak{O}^{f}(\lambda)$. In particular, each module $L^{f}(\mu), \mu \leq \lambda$, has the indecomposable projective cover $I(\mu)$ by [14], Corollary 4.13, and $I(\mu)$ has a Verma composition series: $I(\mu)=I_{0} \supset I_{1} \supset \ldots \supset I_{\ell} \supset 0$, where $I_{i} / I_{i+1} \simeq M^{f}\left(\mu_{i}\right), \mu_{i} \leq \lambda, i=0, \ldots, \ell$, by [14], Corollary 4.10. We denote by $\left(I(\mu): M^{f}(\nu)\right)$ the number of indices $i$ in $\{0, \ldots, \ell\}$ such that $\mu_{i}=\nu$.

Theorem 9.1 (cf. [14], Theorem 6.4). Let $\mu \leq \lambda$ and $\nu \leq \lambda$. Then

$$
\left(I(\mu): M^{f}(\nu)\right)=\left[M^{f}(\nu): L^{f}(\mu)\right]
$$

The main object of our study in this section is the category $\mathfrak{O}_{X}(\lambda)$, the full subcategory of the category of weight $\mathfrak{G}$-modules $V$ such that

1. $P(V) \subset\left\{\mu \in \mathfrak{H}^{*} \mid \lambda-\mu \in Q_{+}\right\}$.

2. $\operatorname{dim} V_{\mu}<\infty$ for all $\mu \leq \lambda$.

3. The module $V$ is generated by $V^{f}=\sum_{\mu \leq \lambda} V_{\mu}$.

4. $\mathfrak{G}_{-}(\tilde{\pi}(\lambda)) v=0$ for all $v \in V^{f}$.

Remark 9.2. 1. A similar category with $\lambda(c) \neq 0$ was introduced and studied for non-twisted affine algebras in [2], and in general in [3].

2. The modules $\check{M}(\lambda), \hat{M}(\lambda)$ and $L_{X}(\lambda)$ are objects of $\mathfrak{O}_{X}(\lambda)$. Moreover, it follows from Theorem 7.7 that any submodule $N \subset \check{M}(\lambda)$ belongs to $\mathfrak{O}_{X}(\lambda)$ as well.

3. If $\lambda$ is not in general position, the category $\mathfrak{O}_{X}(\lambda)$ may not be closed under the operation of taking of submodules. For example, if $(\lambda, \alpha)=0$ for all $\alpha \in \pi$ and $N$ is the maximal $\mathbf{m}$-submodule of $M^{f}(\lambda)$, then $M^{f}(\lambda) / N \simeq \mathbb{C}$ and any $\mathfrak{G}$-submodule of $\check{M}(\lambda) / U(\mathfrak{G}) N$ is not an object of $\mathfrak{O}_{X}(\lambda)$ [7].

4. If $N \in \mathfrak{O}_{X}(\lambda)$ then $N^{f} \in \mathfrak{O}(\lambda)$.

Proposition 9.3. If $V$ is an irreducible module in $\mathfrak{O}_{X}(\lambda)$, then $V \simeq L_{X}(\mu)$ for some $\mu \leq \lambda$.

Proof. Let $V$ be an irreducible module in $\mathfrak{O}_{X}(\lambda)$. Then $V^{f} \in \mathfrak{O}^{f}(\lambda)$ and $V^{f}$ is an irreducible m-module. Thus $V^{f} \simeq L^{f}(\mu)$ for some $\mu \leq \lambda$. Since $P(V) \subset\{\mu \in$ $\left.\mathfrak{H}^{*} \mid \lambda-\mu \in Q_{+}\right\}$, we conclude that $\mathbf{u}_{X}^{+} v=0$ for all $v \in V^{f}$, and hence there exist $\mu \in P(V)$ and a non-zero element $v \in V_{\mu}^{f}$ for which $\mathfrak{G}_{X}^{+} v=0$. This implies that $V$ is a homomorphic image of $\check{M}(\mu)$, and thus $V \simeq L_{X}(\mu)$.

Proposition 9.4. The category $\mathfrak{O}_{X}(\lambda)$ is closed under the operations of taking submodules, quotients and finite direct sums.

Proof. The proof is based on Theorem 7.7, (1) and is analogous to the proof of Corollary 6.6 in [3].

We will prove the equivalence of the categories $\mathfrak{O}_{X}(\lambda)$ and $\mathfrak{O}^{f}(\lambda)$ for $\lambda$ in general position. Define an exact functor $F: \mathfrak{O}_{X}(\lambda) \rightarrow \mathfrak{O}^{f}(\lambda)$ by $F(V)=V^{f}$ and $F(f)=$ $\left.f\right|_{V^{f}}$ for any $V \in \mathfrak{O}_{X}(\lambda)$ and any $f \in \operatorname{Hom}_{\mathfrak{G}}\left(V, V^{\prime}\right)$ in $\mathfrak{O}_{X}(\lambda)$. Also define an exact functor $Y: \mathfrak{O}^{f}(\lambda) \rightarrow \mathfrak{O}_{X}(\lambda)$ as follows. Let $M$ and $M^{\prime}$ be the objects in $\mathfrak{O}^{f}(\lambda)$ and $g \in \operatorname{Hom}_{\mathbf{m}}\left(M, M^{\prime}\right)$. We can make $M$ into a $\mathbf{p}_{X}$-module with a trivial action of $\mathbf{u}_{X}^{+}$ 
and consider a $\mathfrak{G}$-module $Y(M)=\left(U(\mathfrak{G}) / U(\mathfrak{G}) \mathfrak{G}_{-}(\tilde{\pi}(\lambda))\right) \otimes_{U\left(\mathbf{p}_{X}\right)} M \in \mathfrak{O}_{X}(\lambda)$ and $Y(g)=1 \otimes g$. By Corollary 7.9 we immediately conclude that $Y \circ F(\check{M}(\mu)) \simeq \check{M}(\mu)$ and $F \circ Y\left(M^{f}(\mu)\right) \simeq M^{f}(\mu)$ for $\mu \leq \lambda$.

Theorem 9.5. Let $\lambda \in \mathfrak{H}^{*}, \lambda(c)=0$ and $\lambda$ in general position. Then the categories $\mathfrak{O}_{X}(\lambda)$ and $\mathfrak{O}^{f}(\lambda)$ are equivalent.

Proof. The proof is absolutely analogous to the proof of Theorem 6.7 in [3].

For $\mu \leq \lambda$ denote $I_{X}(\mu)=Y(I(\mu))$. Then $I_{X}(\mu)$ is an indecomposable projective cover for $L_{X}(\mu)$ by Theorem 9.5 , and it has a Verma composition series: $I_{X}(\mu) \supset$ $I_{0} \supset I_{1} \supset \ldots \supset I_{\ell} \supset 0, I_{i} / I_{i+1} \simeq \check{M}\left(\mu_{i}\right), \mu_{i} \leq \lambda, i=0, \ldots, \ell$. Let $\left(I_{X}(\mu): \check{M}(\nu)\right)$ be the number of $j$ 's such that $\mu_{j}=\nu$.

Since any object of $\mathfrak{O}^{f}(\lambda)$ has a local composition series [13], it follows from Theorem 9.5 that any object $V \in \mathfrak{O}_{X}(\lambda)$ has a local composition series, and $\left[V: L_{X}(\mu)\right]$ denotes the multiplicity of $L_{X}(\mu)$ in $V$.

Theorem 9.6 (BGG duality). Let $\lambda \in \mathfrak{H}^{*}, \lambda(c)=0, \lambda$ in general position and $\mu \leq \lambda, \nu \leq \lambda$. Then

$$
\left(I_{X}(\mu): \check{M}(\nu)\right)=\left[\check{M}(\nu): L_{X}(\mu)\right] .
$$

Proof. It follows from Theorems 9.5 and 9.1 that $\left(I_{X}(\mu): \check{M}(\nu)\right)=\left(I(\mu): M^{f}(\nu)\right)$ $=\left[M^{f}(\nu): L^{f}(\mu)\right]=\left[\check{M}(\nu): L_{X}(\mu)\right]$.

\section{Generalized Verma type modules of leVel zero}

Let $X \neq I \cup \emptyset, S \subsetneq \pi^{f}, \lambda \in \mathfrak{H}^{*}, \lambda(c)=0$ and $\lambda_{S}$ is dominant integral. Set $\mathfrak{A}=\mathfrak{G}_{-}(\tilde{\pi}(\lambda))$ and consider the $\mathfrak{G}$-submodules $M_{S}=U(\mathfrak{G})\left(\bar{G}_{-} \oplus \mathfrak{A}\right)\left(1 \otimes V_{S}(\lambda)\right)$ and $M_{S}^{\prime}=U(\mathfrak{G}) \mathfrak{A}\left(1 \otimes V_{S}(\lambda)\right)$ of $M_{X, S}(\lambda)$. Let $\hat{M}_{S}(\lambda)=M_{X, S}(\lambda) / M_{S}$ and $\check{M}_{S}(\lambda)=M_{X, S}(\lambda) / M_{S}^{\prime}$. Then both $\hat{M}_{S}(\lambda)$ and $\check{M}_{S}(\lambda)$ are weight $\mathfrak{G}$-modules with the unique irreducible quotient $L_{X}(\lambda)$, and one has the following chain of surjective homomorphisms:

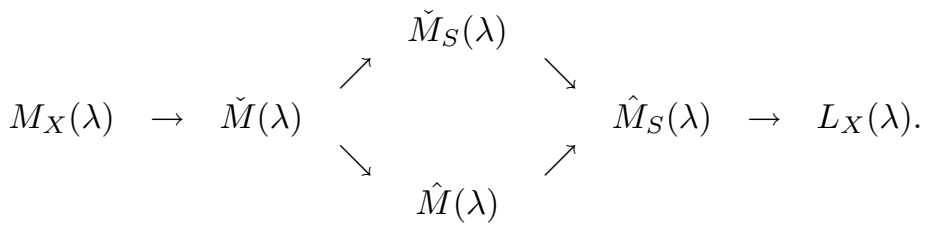

Clearly, $\check{M}_{S}^{f}(\lambda) \simeq M_{X, S}^{f}(\lambda)$ and $\hat{M}_{S}^{f}(\lambda) \simeq M_{X, S}^{f}(\lambda) /\left(M_{X, S}^{f}(\lambda) \cap M_{S}\right)$.

Remark 10.1. If $S=\emptyset$ then $\hat{M}_{\emptyset}(\lambda) \simeq \hat{M}(\lambda)$.

The next statement describes the structure of modules $\hat{M}_{S}(\lambda)$ and $\check{M}_{S}(\lambda)$ with $\lambda$ in general position.

Theorem 10.2. Let $X \neq I \cup \emptyset, S \subsetneq \pi^{f}, \lambda, \mu \in \mathfrak{H}^{*}, \lambda(c)=\mu(c)=0$, both $\lambda$ and $\mu$ in general position and both $\lambda_{S}$ and $\mu_{S}$ dominant integral.

1. If $N \subset \hat{M}_{S}(\lambda)$ (resp. $\left.N \subset \check{M}_{S}(\lambda)\right)$, then $N$ is generated by $N^{f}$ and

$$
N \simeq\left(U(\mathfrak{G} / \mathfrak{U}(\mathfrak{G}) \mathfrak{A}) \otimes_{U\left(\mathbf{p}_{X}\right)} N^{f},\right.
$$

where $u_{X}^{+}$acts trivially on $N^{f}$. 
2. $\operatorname{Hom}_{\mathfrak{G}}\left(\check{M}_{S}(\lambda), \check{M}_{S}(\mu)\right) \simeq \operatorname{Hom}_{\mathbf{m}}\left(M_{X, S}^{f}(\lambda), M_{X, S}^{f}(\mu)\right)$, $\operatorname{Hom}_{\mathfrak{G}}\left(\hat{M}_{S}(\lambda), \hat{M}_{S}(\mu)\right) \simeq \operatorname{Hom}_{\tilde{\mathfrak{G}} f}\left(\hat{M}_{S}^{f}(\lambda), \hat{M}_{S}^{f}(\mu)\right)$.

3. Both $\check{M}_{S}(\lambda)$ and $\hat{M}_{S}(\lambda)$ have local composition series, $\left[\check{M}_{S}(\lambda): L_{X}(\mu)\right]=$ $\left[M_{X, S}^{f}(\lambda): L^{f}(\mu)\right]$ and $\left[\hat{M}_{S}(\lambda): L_{X}(\mu)\right]=\left[\hat{M}_{S}^{f}(\lambda): L^{f}(\mu)\right]$.

Proof. Let $\hat{\tau}_{S}: \hat{M}(\lambda) \rightarrow \hat{M}_{S}(\lambda)$ (resp. $\check{\tau}_{S}: \check{M}(\lambda) \rightarrow \check{M}_{S}(\lambda)$ ) be a natural epimorphism. There exists a submodule $\hat{N} \subset \hat{M}(\lambda)$ (resp. $\check{N} \subset \check{M}(\lambda))$ such that $\hat{\tau}_{S}(\hat{N})=N\left(\operatorname{resp} . \check{\tau}_{S}(\check{N})=N\right)$ and $\hat{\tau}\left(\hat{N}^{f}\right)=N^{f}\left(\right.$ resp. $\left.\check{\tau}_{S}\left(\check{N}^{f}\right)=N^{f}\right)$. Thus, statement (1) follows from Corollary 7.8 and Corollary 7.9. Further, (1) implies that $\check{M}_{S}(\lambda)\left(\right.$ resp. $\left.\hat{M}_{S}(\lambda)\right)$ is generated by $M_{X, S}^{f}(\lambda)\left(\right.$ resp. $\left.\hat{M}_{S}^{f}(\lambda)\right)$, and hence $(2)$ and (3) follow. This completes the proof.

Remark 10.3. Let $X \neq I \cup \emptyset, S \subsetneq \pi^{f}, S$ is connected, $\lambda_{0} \in \mathfrak{H}^{*}$ and $\left(\lambda_{0}, \alpha\right)=0$ for all $\alpha \in \bar{\pi}^{f}$. Using [14], Theorem 9.12 and following [3], Theorem 5.7, one can construct the generalized strong BGG resolution for modules $\hat{M}_{S}\left(\lambda_{0}\right)$. We omit the details.

\section{1. "Truncated" Categories $\mathfrak{O}_{X, S}(\lambda, q)$}

Let $X \neq I \cup \emptyset, S \subsetneq \pi^{f}, \lambda \in \mathfrak{H}^{*}, \lambda(c)=0$ and $q \in \mathbb{Z}_{+}$. Define ${ }_{q} Q^{+}=$ $\left\{\mu=\sum m_{\alpha} \alpha \in Q_{+}^{f} \mid \alpha \in \pi, m_{\alpha} \in \mathbb{Z}_{+}, \sum m_{\alpha}>q\right\}, \Pi=\left\{\mu \in \mathfrak{H}^{*} \mid \mu-\lambda=\right.$ $\left.\sum_{\alpha \in \pi} m_{\alpha} \alpha \in Q^{f}, m_{\alpha} \in \mathbb{Z},(\mu-\lambda)^{+}=\sum_{m_{\alpha} \in \mathbb{Z}_{+}} m_{\alpha} \alpha \in Q_{+}^{f} \backslash_{q} Q^{+}\right\}, \Pi^{S}=\{\mu \in$ $\Pi \mid \mu_{S}$ is integral dominant\}. Clearly, there is a one-to-one correspondence between elements of $\Pi^{S}$ and irreducible finite-dimensional $\mathbf{m}_{S}$-modules $V_{S}(\mu)$ with highest weight $\mu \in \Pi$.

Consider the full subcategory $\mathfrak{O}_{S}^{f}=\mathfrak{O}_{S}^{f}(\lambda, q)$ of the category of weight $\mathbf{m}$ modules, whose objects $V$ satisfy

1. $\operatorname{dim} V_{\mu}<\infty$ for all $\mu \in \mathfrak{H}^{*}$.

2. $V$ is a direct sum of $V_{S}(\mu)^{\prime} s, \mu \in \Pi^{S}$ (cf. [14] and [3]).

The category $\mathfrak{O}_{S}^{f}$ is stable under the operations of taking submodules, quotients and finite direct sums. Note that $M_{X, S}^{f}(\mu)$ and $L^{f}(\mu)$ belong to $\mathfrak{O}_{S}^{f}$ for all $\mu \in \Pi^{S}$, and the modules $L^{f}(\mu), \mu \in \Pi^{S}$, exhaust all irreducible objects in $\mathfrak{O}_{S}^{f}$.

Fix $\mu \in \Pi^{S}$ and set $D_{X, S}=\mathbf{u}_{X, S}^{+} \oplus \bar{G}_{+}, U\left(D_{X, S}\right)^{(\mu)}=\sum U\left(D_{X, S}\right)_{\alpha}, \alpha \in Q$, $\nu+\alpha \notin \Pi$ for all $\nu \in P\left(V_{S}(\mu)\right)$. Then $\mathfrak{A}(\mu, S)=\left(U\left(D_{X, S}\right) / U\left(D_{X, S}\right)^{(\mu)}\right) \otimes V_{S}(\mu)$ is a weight $\mathbf{p}_{X, S} \oplus \bar{G}_{+}$-module where $D_{X, S}$ acts on the left and $\mathbf{m}_{S}$ by the tensor product action. Let $\bar{A}(\mu, S)=\sum A(\mu, S)_{\nu}, \nu \in \mathfrak{H}^{*} \backslash \Pi$, and

$$
\tilde{\mathfrak{A}}(\mu, S)=\mathfrak{A}(\mu, S) / U\left(\mathbf{p}_{X, S} \oplus \bar{G}_{+}\right) \overline{\mathfrak{A}}(\mu, S) .
$$

Then $P^{S}(\mu)=U(\mathbf{m}) \otimes_{U\left(\mathbf{p}_{X, S} \oplus \bar{G}_{+}\right)} \tilde{\mathfrak{A}}(\mu, S)$ is a projective object in $\mathfrak{O}_{S}^{f}$. Again, by [14], Corollary 4.13, there is a one-to-one correspondence between the irreducible objects in $\mathfrak{O}_{S}^{f}$ and the indecomposable direct summands of the $P^{S}(\mu)$ 's, $\mu \in \Pi^{S}$. Let $I^{S}(\mu)$ be the indecomposable projective cover for $L^{f}(\mu)$.

By [14], Corollary 4.10, the module $I^{S}(\mu)$ has a generalized Verma composition series, i.e. there exists a filtration $I^{S}(\mu)=I_{0} \supset I_{1} \supset \ldots \supset I_{\ell} \supset 0$ where $I_{i} / I_{i+1} \simeq$ $M_{X, S}^{f}\left(\mu_{i}\right), \mu_{i} \in \Pi^{S}$. Denote by $\left(I^{S}(\mu): M_{X, S}^{f}(\nu)\right)$ the number of $i$ 's such that $\nu=\mu_{i}$. 
Theorem 11.1 (cf. [14], Theorem 6.4). If $\mu, \nu \in \Pi^{S}$, then $\left(I^{S}(\mu): M_{X, S}^{f}(\nu)\right)=$ $\left[M_{X, S}^{f}(\nu): L^{f}(\mu)\right]$.

Now suppose that $\lambda$ is in general position, and consider the full subcategory $\mathfrak{O}_{X, S}=\mathfrak{O}_{X, S}(\lambda, q)$ of the category of weight $\mathfrak{G}$-modules $V$ such that

1. $P(V) \subset \cup_{\mu \in \Pi}\left\{\nu \in \mathfrak{H}^{*} \mid \mu-\nu \in Q_{+}\right\}$.

2. $\operatorname{dim} V_{\mu}<\infty$ for all $\mu \in \Pi$.

3. The module $V$ is generated by $V^{f}=\sum_{\mu \in \Pi} V_{\mu}$.

4. $V^{f}$ is a direct sum of $V_{S}(\mu)^{\prime}$ 's, $\mu \in \Pi^{S}$.

5. $\mathfrak{G}_{-}(\tilde{\pi}(\lambda)) v=0$ for all $v \in V^{f}$.

Remark 11.2. 1. If $\mu \in \Pi^{S}$, then the modules $\check{M}_{S}(\mu), \hat{M}_{S}(\mu)$ and $L_{X}(\mu)$ are objects of the category $\mathfrak{O}_{X, S}$.

2. If $V \in \mathfrak{O}_{X, S}$ then $V^{f} \in \mathfrak{O}_{S}^{f}$.

3. If $V \in \mathfrak{O}_{X, S}$ is irreducible then $V \simeq L_{X}(\mu)$ for some $\mu \in \Pi^{S}$. The proof is analogous to the proof of Proposition 9.3.

4. The category $\mathfrak{O}_{X, S}$ is closed under the operations of taking submodules, quotients and finite direct sums (cf. Proposition 3.4).

5. $\mathfrak{O}_{X, \emptyset}(\lambda, 0)=\mathfrak{O}_{X}(\lambda)$.

Theorem 11.3. Let $\lambda \in \mathfrak{H}^{*}, \lambda(c)=0, \lambda$ in general position and $q \in \mathbb{Z}_{+}$. Then the categories $\mathfrak{O}_{X, S}(\lambda, q)$ and $\mathfrak{O}_{S}^{f}(\lambda, q)$ are equivalent.

Proof. As in section 9 define exact functors $F: \mathfrak{O}_{X, S} \rightarrow \mathfrak{O}_{S}^{f}$ and $Y: \mathfrak{O}_{S}^{f} \rightarrow \mathfrak{O}_{X, S}$. Then $Y \circ F\left(\check{M}_{S}(\mu)\right) \simeq \check{M}_{S}(\mu)$ and $F \circ Y\left(M_{X, S}^{f}(\mu)\right) \simeq M_{X, S}^{f}(\mu)$ for all $\mu \in \Pi^{S}$ by Theorem 10.2. Moreover, $F$ and $Y$ establish an equivalence of $\mathfrak{O}_{X, S}$ and $\mathfrak{O}_{S}^{f}$. The proof follows the proof of Theorem 6.7 in [3].

For $\mu \in \Pi^{S}$ let $I_{X}^{S}(\mu)=Y\left(I^{S}(\mu)\right)$. Then $I_{X}^{S}(\mu)$ is an indecomposable projective cover for $L_{X}(\mu)$ in $\mathfrak{O}_{X, S}$ by Theorem 11.3, and it has a generalized Verma composition series with factors $\check{M}_{S}\left(\mu_{i}\right), \mu_{i} \in \Pi^{S}$. Denote by $\left[I_{X}^{S}(\mu): \check{M}_{S}(\nu)\right]$ the multiplicity of $\check{M}_{S}(\nu)$ in a generalized Verma composition series for $I_{X}^{S}(\mu)$ and by $\left(\check{M}_{S}(\nu): L_{X}(\mu)\right)$ the multiplicity of $L_{X}(\mu)$ in a local composition series for $\check{M}_{S}(\nu)$.

Theorem 11.4 (BGG duality). If $\lambda$ is in general position and $\mu, \nu \in \Pi^{S}$, then

$$
\left[I_{X}^{S}(\mu): \check{M}_{S}(\nu)\right]=\left(\check{M}_{S}(\nu): L_{X}(\mu)\right) .
$$

Proof. Follows from Theorems 11.3 and 11.1.

\section{Some Subcategories of $\mathfrak{O}_{X, S}(\lambda, q)$}

Consider the full subcategory $\overline{\mathfrak{O}}_{S}^{f}=\overline{\mathfrak{D}}_{S}^{f}(\lambda, q) \subset \mathfrak{O}_{S}^{f}(\lambda, q)$ consisting of mmodules $V$ such that $\bar{G} v=0$ for all $v \in V$, and the full subcategory $\overline{\mathfrak{O}}_{X, S}=$ $\overline{\mathfrak{D}}_{X, S}(\lambda, q) \subset \mathfrak{O}_{X, S}(\lambda, q)$ consisting of $\mathfrak{G}$-modules $M$ such that $M^{f} \in \overline{\mathfrak{O}}_{S}^{f}$. Obviously, $\hat{M}_{S}(\mu)$ and $L_{X}(\mu)$ are objects of $\overline{\mathfrak{D}}_{X, S}$ for any $\mu \in \Pi^{S}$.

Let $\mu \in \Pi^{S}$. If we replace $\bar{G}_{+}$by $\bar{G}$ in the construction of $P^{S}(\mu)$ we obtain a projective module $\bar{P}^{S}(\mu)$ in $\overline{\mathfrak{D}}_{S}^{f}$, whose indecomposable summands exhaust all indecomposable projectives in $\overline{\mathfrak{O}}_{S}^{f}$. Let $\bar{I}^{S}(\mu)$ be the indecomposable projective cover for $L^{f}(\mu)$. 
Since $F(M) \in \overline{\mathfrak{O}}_{S}^{f}$ for any $M \in \overline{\mathfrak{D}}_{X, S}$ and $Y(N) \in \bar{O}_{X, S}$ for any $N \in \overline{\mathfrak{D}}_{S}^{f}$, the functors $F$ and $Y$ induce the exact functors $\bar{F}: \overline{\mathfrak{O}}_{X, S} \rightarrow \overline{\mathfrak{O}}_{S}^{f}$ and $\bar{Y}: \overline{\mathfrak{O}}_{S}^{f} \rightarrow \overline{\mathfrak{O}}_{X, S}$. Denote $\bar{I}_{X}^{S}(\mu)=Y\left(I^{S}(\mu)\right)$, the indecomposable projective cover for $L_{X}(\mu)$ in $\overline{\mathfrak{O}}_{X, S}$.

Theorem 12.1. Let $\mu, \nu \in \Pi^{S}(\lambda, q)$.

1. $\left[\bar{I}^{S}(\mu): \hat{M}_{S}^{f}(\nu)\right]=\left(\hat{M}_{S}^{f}(\nu): L^{f}(\mu)\right)$.

2. If $\lambda$ is in general position, then the categories $\overline{\mathfrak{D}}_{X, S}$ and $\overline{\mathfrak{O}}_{S}^{f}$ are equivalent.

3. If $\lambda$ is in general position, then

$$
\left[\bar{I}_{X}^{S}(\mu): \hat{M}_{S}(\nu)\right]=\left(\hat{M}_{S}(\nu): L_{X}(\mu)\right)
$$

Proof. The proof of (1) follows the general lines of [14], Theorem 6.4; the proof of (2) is analogous to the proof of Theorem 6.7 in [3]; and (3) follows from (1) and (2).

\section{REFERENCES}

1. B. Cox, Verma modules induced from nonstandard Borel subalgebras, Pacific J. Math. 165 (1994), 269-294. MR 95m:17017

2. B. Cox, Structure of the non-standard category of highest weight modules, Queen's Papers in Pure and Applied Math. 94 (1994), 35-47. MR 95d:17026

3. B. Cox, V. Futorny, D. Melville, Categories of nonstandard highest weight modules for affine Lie algebras, Math. Z. 221 (1996), 193-209. CMP 96:08

4. V. Doedhar, O. Gabber, V. Kac, Structure of some categories of representations of infinite dimensional Lie algebras, Adv. in Math. 45 (1982), 92-116. MR 83i:17012

5. V. Futorny, Parabolic partitions of root systems and corresponding representations of affine Lie algebras, Preprint No. 1990-8, Inst. Math. Acad. Sci. Ukraine, Kiev, 1990, pp. 30-39. MR 92a: 17036

6. V. Futorny, The parabolic subsets of root systems and corresponding representations of affine Lie algebras, Contemp. Math. 131 (1992), 45-52. MR 93b:00030

7. V. Futorny, Imaginary Verma modules for affine Lie algebras, Canad. Math. Bull., 37 (1994), 213-218. MR 95a:17030

8. V. Futorny, Verma type modules over affine Lie algebras, Funkts. Anal. i ego Prilozhen. 27 (1993), no. 3, 92-94; English transl., Funct. Anal. Appl. 27 (1993), 224-225. CMP 96:05

9. V. Futorny, H. Saifi, Modules of Verma type and new irreducible representations for affine Lie algebras, CMS Conference Proc. 14 (1993), 185-191. MR 94j:16002

10. H.P. Jakobsen, V.G. Kac, A new class of unitarizable highest weight representations of infinite-dimensional Lie algebras, Lecture Notes in Physics, 226 (1985), 1-20. MR 87g:17020

11. H.P. Jakobsen, V.G. Kac, A new class of unitarizable highest weight representations of infinite-dimensional Lie algebras, II, J. Funct. Anal. 82 (1989), 69-90. MR 89m:17032

12. V. Kac, Infinite dimensional Lie algebras, Cambridge University Press, third edition, 1990. MR 92k:17038

13. R. Moody, A. Pianzola, Lie algebras with triangular decomposition, Wiley, 1995. MR 96d:17025

14. A. Rocha-Caridi, N. Wallach, Projective modules over graded Lie algebras, Math. Z. 180 (1982), 151-177. MR 83h:17018

Department of Mathematics, Kiev University, Kiev, Ukraine 252033

E-mail address: futorny@uni-alg.kiev.ua 